\title{
Cell atlas of human uterus
}

Bingbing $\mathrm{Wu}^{1,2,3, \#}, \mathrm{Yu} \mathrm{Li}^{1,2,3, \#}$, Yanshan $\mathrm{Liu}^{1,2,3}$, Kaixiu $\mathrm{Jin}^{4}$, Kun $\mathrm{Zhao}^{2,3}$, Chengrui $\mathrm{An}^{2,3}$, Qikai $\mathrm{Li}^{5}$, Lin Gong ${ }^{2,3}$, Wei Zhao ${ }^{6}$, Jinghui $\mathrm{Hu}^{6}$, Jianhua Qian ${ }^{6}$, HongWei Ouyang ${ }^{2,3,7^{*}}$, XiaoHui Zou ${ }^{1,2,3, *}$

${ }^{1}$ Clinical Research Center, the First Affiliated Hospital, School of Medicine, Zhejiang University

${ }^{2}$ Dr.Li Dak Sum and Yip Yio Chin Center for Stem Cell and Regeneration Medicine, Zhejiang University, Hangzhou, Zhejiang 310003, PR China

${ }^{3}$ Zhejiang Provincial Key Laboratory of Tissue Engineering and Regenerative Medicine, Hangzhou, Zhejiang 310058, PR China

${ }^{4}$ School of Mathematical Sciences, Zhejiang University, Hangzhou, 310058, PR China ${ }^{5}$ College of Agriculture \& Biotechnology, Zhejiang University, Hangzhou, 310058, PR China

${ }^{6}$ Department of Gynecology, the First Affiliated Hospital, School of Medicine, Zhejiang University, Hangzhou, Zhejiang 310003, PR China

${ }^{7}$ Zhejiang University-University of Edinburgh Institute, Hangzhou, 310058, PR China

${ }^{\#}$ Co-first author

*Corresponding author

Correspondence and requests for materials should be addressed to X.H.Z. (Email: zouxiaohui@zju.edu.cn)

Corresponding address: Clinical Research Center, the First Affiliated Hospital, School of Medicine, Zhejiang University, 79 Qing Chun Road, Hangzhou, Zhejiang, P.R.

China, 310003, Fax/Phone: +086-0571-88208262 


\section{SUMMARY:}

The human uterus is a highly dynamic tissue that undergoes repeated damage repair and regeneration during the menstrual cycle, which make it ideal model to study tissue regeneration and pathological process. Stem/progenitors were speculated to be involved in the regeneration of endometrial epithelial and pathogenesis of endometriosis. But the identity, microenvironment and regulatory mechanisms of the uterus epithelial stem/progenitors in vivo remain unclear. Here, we dissected the cell heterogeneities of the full-thickness human uterus epithelial cells (11 clusters), stroma cells (6 clusters), endothelial cells (5 clusters), smooth muscle cells (2 clusters), myofibroblasts ( 2 clusters) and immune cells (6 clusters) from 2735 single cell by single cell RNA-seq. Further analysis identified a unique ciliated epithelial cell cluster showing characteristics of stem/progenitors with properties of epithelial-mesenchymal transition (EMT) that mainly localized in the upper functionalis of the endometrium. Ordering the cell subpopulations along the pseudo-space revealed cell clusters possess cellular states of stress, inflammation and apoptosis in the upper functionalis cellular ecosystem of the endometrium. Connectivity map between the human uterus subpopulations revealed potential inflammatory (cytokines and chemokines) and developmental (WNT, FGF, VEGF) signals within the upper functionalis cellular ecosystem of the endometrium, especially from other epithelial clusters, regulating cell plasticity of the EMT-epithelial clusters. This study reconstructed the heterogeneities, space-specific distribution and connectivity map of human uterus atlas, which would provide insight in the regeneration of uterus endometria and reference for the pathogenesis of uterus.

\section{KEYWORDS:}

Uterus; atlas; single cell RNA-Seq; EMT; epithelia 


\section{INTRODUCTION:}

The human uterus comprises of endometrium, myometrium and the blood vessels. It exhibits remarkable plasticity, the endometria would undergo repeated damage and regeneration, the myometrium would enlarge during pregnancy and return to normal size after gravidity(Ono et al.2007). Its highly dynamic properties of repeated injury and scar-less repair along the menstrual cycle make it ideal model to study tissue regeneration and pathological process(Maybin and Critchley 2015).

After the proliferative period of the menstrual cycle, the secretory endometria would have two destinies: either to prepare for implantation by fertilized embryos or to shed (Gargett, Nguyen, and Ye 2012; Cakmak and Taylor 2011). The receptive state of the secretory endometria is vital for the proper implantation of embryo, and abnormal physiological conditions would cause miscarriage(Cakmak and Taylor 2011). On the other hand, the shedding endometrial pellets of the secretory endometria would lead to serious pathological outcomes under certain circumstances, like endometriosis, when the shedding endometrial pellets go reversely along the oviduct into the abdominal cavity(Hufnagel et al. 2015). However, the precise regulatory mechanisms for the preparation of the secretory uterus for embryo implantation and etiology of endometriosis remains unclear(Hufnagel et al. 2015).

Endometrial stem/progenitor cells were shown to be involved in the regeneration of the damaged functional layer during the menstrual cycle (Gargett, Nguyen, and Ye 2012) and continuous growth upon embryo implantation, as well as involved in the pathological process of endometriosis(Hufnagel et al. 2015). However, the precise identify, function and regulatory mechanisms of the uterus epithelial stem/progenitors during these physiological and pathological circumstances in vivo remains unclear(Valentijn et al. 2013; Gargett, Schwab, and Deane 2016).

Tissue microenvironment was shown to be indispensable during the tissue development(Camp et al. 2017), homeostasis(Zepp et al. 2017), regeneration and pathological progression(Puram et al. 2017). Single cell analysis has been increasingly utilized to dissect cell heterogeneity and study the dynamic microenvironment between cell subpopulations during biological process such as 
development, cancer metastasis, tissue homeostasis and pathology(Camp et al. 2017; Puram et al. 2017; Zepp et al. 2017) . Thus, in this study, we reconstructed cell atlas of the secretory phase of human uterus through dissecting the cell heterogeneities, reconstructing spatial distribution and connectivity map of the cell clusters by single cell RNA-seq.

\section{RESULTS:}

\section{Single cell RNA-seq of full-thickness secretory human uterus}

First, we used drop-seq (10x Genomics) based single cell RNA-seq to profile single cell suspension from a full-thickness secretory human uterus tissue (Fig 1A). Then we used the Cell Ranger Pipeline (10x Genomics) to analyze the unique molecular tagged (UMI) Of the raw sequencing data (Fig S1A). We profiled 2735 individual cell of the human uterus (Fig S1B). We obtained saturated sequencing with about 680k reads per cell (Fig S1C), collected 2735 cells with high UMI counts, and the median gene number detected per cell was about 3,183 (Fig S1D).

Unsupervised clustering based on principal components of the most variable expressed genes (Satija et al. 2015) partitioned all the cells into 15 clusters, which we visualized using t-distributed stochastic neighbourhood embedding (t-SNE) (Fig 1B) and principal component analysis (PCA) (Fig S1E, S1F), each cluster possesses a unique set of signature genes (Fig 1C). As labeled by known marker genes, there are clusters of endometrial epithelia, stroma, endothelia, SMA+, immune cells in human uterus. Endometrial epithelial cells express high level of KRT8, KRT18, EPCAM and CLDN3, endometrial stromal cells express high level of MME, FN1, COL3A1, HOXA10, endothelial cell express high level of CD34, VWF, CLDN5, SOX18, SMA+ cells express high level of ACTA2, MYH11, MYL6, MYL9. Endometrial immune cell express high level of PTPRC, CD68, CD163 and CD96 (Fig 1D).

To further investigate cellular heterogeneity of each cluster of the full-thickness human uterus, we cluster the endometrial epithelia, stroma, endothelia, SMA+, immune cells separately, and we acquired 11 sub-clusters of endometrial epithelial cells, 5 sub-clusters of endmotrial stromal cells, 5 sub-clusters of SMA+ cells, 5 
sub-clusters of endothelial cells, 3 sub-clusters of macrophages and 3 sub-clusters of natural killer cells (Fig 1E). Totally, we found 32 sub-clusters from 5 main groups.

\section{Heterogeneity of uterus epithelia cells}

Analysis of each unique signature genes of endometrial epithelial sub-clusters (Fig 2A, 2B) showed heterogeneity of ciliated and secretory epithelial sub-clusters. There are 5 ciliated epithelial sub-clusters (Epi4, Epi5, Epi6, Epi9, Epi10) as labelled by ciliated marker alpha-tubulin expression (TUBA1A and TUBA1B) (Fig 2C). There are 7 secretory epithelial sub-clusters (Epi0, Epi1, Epi2, Epi3, Epi7, Epi8, Epi9) in the uterus as labelled by secretory marker secretoglobin family (SCGB1D4, SCGB2A1) and inflammatory cytokines and chemokines (CXCL8, VEGFA) (Fig 2D). There is a sub-cluster of cells (Epi9) that express both the ciliated epithelial marker (TUBA1A) and secretory epithelial markers (SCGB1D4, SCGB2A1) (Fig 2C,2D).

Interestingly, we found a sub-cluster of ciliated epithelial cells (Epi10) that possess epithelial-mesenchymal transition (EMT) characteristics, which express both epithelial markers (KRT8) and stroma cell markers (COL3A1), as well as EMT transcription factors (SNAI2, ZEB1) (Fig 2E), which were further confirmed by immunofluorescent staining that the EMT epithelial cells were mainly localized in the upper layer of the functionalis of the endometria (Fig 2F). This EMT sub-cluster express low level of estrogen receptor (ESR1) and high level of CD44 (Fig 2E) (a previous reported mouse endometrial stem cell marker(Janzen et al. 2013)). Gene ontology (GO) analysis of the highly expressed genes in this EMT sub-cluster showed enrichment of GO terms associated with morphogenesis and development of epithelia and gland (Fig 2G). All this imply the uniqueness and association of this EMT sub-cluster with endometrial epithelial stem/progenitor cells.

In order to reconstruct the spatial distribution of the epithelial sub-clusters, we conducted monocle (Satija et al. 2015) to align the 11 subpopulations along the pseudo-space (Fig S2A). Results showed that cluster Epi10, Epi6, Epi4 and Epi5 distributed at the beginning of the pseudo-space, while cluster Epi8, Epi7 and Epi9 distributed at the end of the pseudo-space (Fig S2B). GO analysis with the highly 
expressed genes of cluster Epi10, Epi6, Epi4 and Epi5 showed enrichment of GO terms of cell cycle and DNA synthesis (Fig 2G, Fig S3C-E). GO analysis with the highly expressed genes of cluster Epi2, Epi3, Epi7 and Epi8 showed enrichment of GO terms of response of hypoxia, regulation of apoptotic process and interferon signaling pathway (Fig S3A, S3B, S3F, S3G). These results indicated a ciliated-secretory distribution pattern of the epithelial cells along the uterus cavity (Fig 2H).

\section{Heterogeneity of uterus stroma cells}

Further clustering the stroma cells of uterus revealed 6 distinct cell populations with their unique molecular signatures (Fig 3A, 3B). In order to reconstruct the spatial distribution of the stroma cell, we conducted monocle to align the 6 subpopulations along the pseudo-space (Fig S4A). Results showed that cluster Stro2, Stro3 and Stro4 distributed at the beginning of the pseudo-space, while cluster Stro0, Stro1 and Stro5 distributed at the end of the pseudo-space (Fig S4B). GO analysis with the highly expressed genes of cluster Stro2 and Stro3 showed enrichment of GO terms of cell adhesion, cell differentiation and regulation of wound healing (Fig 3D, 3E ,3F). These results indicated that the cluster Stro2, Stro3 and Stro4 were corresponding to stroma cells from the basal layer of the endometrial, which was responsible for the cyclic regeneration of the shedded functional layer. GO analysis with the highly expressed genes of cluster Stro0, Stro1 and Stro5 showed enrichment of GO terms of response of hypoxia and regulation of apoptotic process (Fig 3C, 3G). These results implied that the cluster Stro0, Stro1 and Stro5 were corresponding to stroma cells from the functional layer of the endometrial, which was far away from the blood vessels and would undergo apoptosis upon shedding during each menstrual cycle. These results indicated a basal-functional distribution pattern of the stroma cells along the uterus cavity (Fig 3H).

\section{Heterogeneity of uterus endothelia cells}

Blood vessels were vital for the regeneration of the uterus endometria, and the 
endothelia cells would also undergo repeated shedding and regeneration during each menstrual cycle(Maybin and Critchley 2015). Further clustering the endothelia cells of uterus revealed 5 distinct cell populations with their unique molecular signatures (Fig 4A, 4B, 4C). In order to reconstruct the spatial distribution of the endothelia cell, we conducted monocle to align the 5 subpopulations along the pseudo-space (Fig S5A). Results showed that the cluster Endo4, Endo3 and Endo1 distributed at the beginning of the pseudo-space, while the cluster Endo2 and Endo0 located at the end of the pseudo-space (Fig S5B). GO analysis revealed that genes associated with T cell co-stimulation and antigen processing and presentation were highly expressed in cluster Endo2 (Fig 4F). Genes associated with angiogenesis and endothelial cell differentiation were highly expressed in cluster Endo1 and Endo4 (Fig 4E,4G). These results also indicated a basal-functional distribution pattern of the endothelia along the uterus cavity (Fig 4H). As the functional region of the endothelia would shed during the menstrual phage, which was mainly mediate by immune cells, and the basal region of the endothelia would be responsible for the regeneration of the blood vessel in the functional layer.

\section{Heterogeneity of uterus smooth muscle and myofibroblasts}

Further clustering the SMA+ cells of uterus revealed 5 distinct cell populations with their unique molecular signatures (Fig 5A, 5B). We identified 2 clusters of smooth muscles (SMA1 \& SMA3), which expressed high level of ACTG2, ACTA2 and DES (Fig 5C). We also found 2 clusters of myofibroblasts (SMA0, SMA4), which expressed high level of COL4A1 and ACTA2 (Fig 5C). Both of the myofibroblasts were in stress (Fig 5D) or inflammatory states (Fig 5G) according to their GO analysis of their highly expressed signature genes. Myofibroblasts were involved in the cyclic menstrual injury and scarless regeneration through wound contraction and synthesis and remodeling of ECM (Maybin and Critchley 2015). Excessive activation of myofibroblasts caused by abnormal regulations were highly correlated with adenomyosis(Ibrahim et al. 2017) and endmetriosis (van Kaam et al. 2008) through abnormal deposition of extracellular matrix from myofibroblast, the discovery of its 
dysregulation factors may shed light on the pathogenesis of these diseases(Liu et al. 2016).

\section{Heterogeneity of uterus immune cells}

Immune cells were highly correlated with physiology and pathology of the uterus(Cousins et al. 2016; Fu et al. 2017). Unsupervised clustering of the immune cells in human uterus revealed 3 sub-cluster of macrophages and 3 cub-clusters of natural killer cells (Fig 6A), as labeled by the expression of CD86 and CD96 (Fig 6B), respectively, each sub-cluster of macrophage (Fig 6C) and natural killer cells (Fig 6D) possess their unique molecular signatures. The 3 macrophage clusters showed distinct expression of functional markers (CSF1R and TLR4, Fig 6E). NK cell subsets were reported to rebuild and maintain appropriate local microenvironment for fetal growth during early pregnancy(Fu et al. 2017). Distinct subsets of monocytes/macrophages were spatio-temporally distributed, responsible for breakdown, repair and remodeling, respectively(Cousins et al. 2016). Abnormal interactions between the immune cells and the endometrial tissues were highly correlated with endometriosis, the abnormal NK cell activity would cause inadequate removal of menstrual debris and macrophages would further facilitate the proliferation of the menstrual debris in peritoneal cavity (Seli and Arici 2003).

\section{Stress, inflammatory and apoptotic ecosystem of the uterus endometria}

The uterus forms unique compartmentalized econsystem of stress, inflammation and apoptotic, which contain epithelial subpopulations (Epi 2, 3, 4, 6, 7, 8, 10), stroma cell subpopulations (Stro0, 1, 5), endothelial cell subpopulations (Endo 0, 2), myofibroblasts (SMA 0,4) and immune cells (macrophage 1, 2, 3 and NK cell 1,2,3), according to the pseudo-space (Fig S4, S5) and GO analysis (Fig S3, 3-5). We next verified these results by using immune fluorescence. The results showed that the upper functionalis layer of the endometria was surrounded by more CD45+ lymphocyte compared with those of the basalis and myometrial layer of the uterus (Fig 7A). While the CD68+ macrophages were evenly distributed in the three layer of 
the uterus (Fig 7B). We use TUNEL kit to detect the number of apoptotic cells in the full-thickness uterus. There are more TUNEL positive staining in the upper functionalis compared with those of the basalis and myometrial layer of the uterus (Fig 7C). Next we use $\gamma \mathrm{H} 2 \mathrm{~A} . \mathrm{X}$ as marker of DNA damage marker, and we found that there are abundant $\gamma \mathrm{H} 2 \mathrm{~A} . \mathrm{X}$ positive staining in the upper functionalis, even in the some of the epithelial cells, while there's hardly any $\gamma \mathrm{H} 2 \mathrm{~A} . \mathrm{X}$ positive cells in the basalis and myometrial layer of the uterus (Fig 7E). Our results confirmed that there was a compartmentalized, stress, inflammatory and apoptotic ecosystem in the upper layer of the functionalis of the uterus endometria.

\section{Connectivity map of human uterus cells reveal microenvironment regulating} epithelial plasticity

As different cell subpopulations were physically surrounded by each other, communications among cells would regulate cell state and even determine cell fate(Camp et al. 2017; Zepp et al. 2017). Thus, we reconstructed the intra-uterus connectivity map among subpopulations by using known ligand-receptor pairs. Finally, we get 1024 connections of 2000 ligand-receptor pairs from 32 sub-clusters (Fig 8A).

Nest we studied the potential regulatory microenvironment of the epi10 subpopulation with EMT properties using the connectivity map. As the epi10 cell population localized at the upper layer ecosystem of the functionalis of the uterus (Fig 8B) with the common characteristics of stress, inflammation and apoptotic. Connectivity map of the epi10 revealed that all the epithelial cell subpopulations showed more ligand-receptor pair interactions to epi10 compared to those of the other cell populations (Fig 8B). Further analysis of the ligands secreted into the microenvironment of the epi10 showed abundant extracellular matrix (LAM, FN1, COL), growth factors (FGF, WNT, BMP, VEGF,), inflammatory cytokines (C3, TNF, CXCL, IL12, CCL2) (Fig 8C), which showed enrichment of GO terms of inflammatory response, WNT signaling, epithelial cell proliferation, mesoderm formation and epithelial to mesenchymal transition (Fig 8D). These results implied 
that the fate of the EMT epithelial cluster was regulated and reprogrammed by its microenvironment, which may reprogram other differentiated epithelial cells into the EMT cluster.

\section{DISCUSSION:}

Heterogeneity and cross-talk among subpopulations of complex tissues and organs regulate development. homeostasis, regeneration and pathology(Camp et al. 2017; Zepp et al. 2017; Puram et al. 2017), which remain great challenges until the wide-spread applications of single cell RNA-seq. Here, we reconstructed an atlas of the human uterus tissues: Our atlas provided the most detailed cell diversity of the uterus tissue so far. Our atlas provided a compartmentalized cell ecosystem. Our atlas highlighted a EMT program in the epithelial subset. Our atlas provided a dynamic connectivity map of the uterus with diverse communications in the upper functionalis layer of the endometria regulating the EMT program.

\section{Our atlas provided the most detailed cell diversity of the uterus tissue}

Most of previous study on uterus biology were based on bulk uterus/endometrium tissue transcriptomics analysis(Diaz-Gimeno et al. 2014) or comparison between different region of the tissue(Evans et al. 2014). As the advance of technology and development analysis pipelines, studies have come to the single cell level(Proserpio and Lonnberg 2016). One of our previous study on uterus epithelial development was based on single cell analysis(Wu et al. 2017). A previous report compared the transcriptomics of endometrial stroma cells in vivo and in vitro by single cell RNA-seq (Krjutskov et al. 2016). We found 32 functional distinct sub-clusters from 5 main groups of the full-thickness uterus tissues in total. Our atlas thus provided the most detailed cell diversity of the uterus tissue so far.

\section{Our atlas provided a compartmentalized cell ecosystem}

We discovered subsets of endmotrial epithelial, stromal, endothelial cells and myofibroblasts with distinct states, some subsets showed state of proliferation, while 
others were in hypoxia, stress and inflammatory states, the rest were in states of development, wound healing and regeneration states. The hypoxia and stress were reported to be functional for the angiogenesis, proliferation and metabolism during the menstrual cycle of the uterus that resemble the process of ischemia and reperfusion (Maybin and Critchley 2015).

\section{Our atlas highlighted a EMT program in the epithelial subset of the uterus}

Our study discovered an unique subset of epithelial cells of the uterus with stem/progenitor property of proliferation, EMT and low hormone receptor expression, which was similar to the characteristics of the epithelial cell cluster we previous reported during the development of mice uterus that was also highly proliferative, EMT and low hormone receptor expression and located in the luminal layer of the uterus (Wu et al. 2017).

Previous reports hypothesized that potential human endometrial epithelial stem/progenitor cells were mainly located in the gland of the basalis layer of the endometrium (Gargett 2007; Nguyen, Sprung, and Gargett 2012). SSEA1 positive cells were shown to possess some stem/progenitor cell properties (longer telomerase activities, more quiescent and lower expresion of estrogen receptor) in vitro, which mainly located in the basalis gland of human endometria (Valentijn et al. 2013). Though mice quiescent epithelial label retaining cells were reported to be located mainly in the luminal epithelia and did not express the estrogen receptor (Chan and Gargett 2006), but their quiescence determined their difference with our proliferative EMT subset. The EMT epithelial cell subset in our study expressed higher level of CD44, a marker of the mouse endometrial epithelial progenitors located at both lumen and gland adjacent to the lumen that also express low level of hormone receptors (Janzen et al. 2013). However, the EMT epithelial cell subset in our study did not express SOX9, transcription factor reported to be vital for the regulation of endometrial epithelial stem/progenitor cells in the basal glands(Valentijn et al. 2013). Thus, our atlas provided a novel stem/progenitor cell subtype.

Cell plasticity was involved in the tissue development, homeostasis maintenance 
and pathological conditions (Varga and Greten 2017). Cell plasticity was increasingly reported in injury and regeneration of various epithelial tissues (e.g. intestine(Tetteh et al. 2016), liver, pancreas(Tritschler et al. 2017), hair follicles(Merrell and Stanger 2016)) in vivo. Endometrial cells were also reported to be highly plastic(Bilyk et al. 2017). Evidences already exist that EMT and MET involved in the menstrual regeneration and embryo implantation in the uterus(Bilyk et al. 2017). Subpopulation of MET cells in the regeneration zone that express both epithelial marker cytokeratin and stromal cell marker vimentin was involved in the menstrual endometrial regeneration (Patterson et al. 2013). MET of endometrial cells would make them more susceptible to deeper penetration by the embryo as epithelial cell gradually lose polarity and tight junctions (Paria et al. 1999).

EMT is also shown to properties of endometrial epithelial stem/progenitor cells(Wu et al. 2017), which may explain the in vitro culturing of endometrial epithelial stem/progenitor cells from menstrual shedding debris since 10 years ago(Gargett, Schwab, and Deane 2016). EMT was also involved in the pathogenesis, during which, epithelial cells would lose polarity and acquire motility, migration and proliferation, which would play a role in the evolution of adenomyosis, endometriosis and even cancer when dysregulated or under certain microenvironment(Bilyk et al. 2017).

\section{Our atlas provided a dynamic connectivity map of the uterus with diverse communications in the upper functionalis layer of the endometria regulating the EMT program}

The low expression of hormone receptor in the reported stem/progenitor cells of SSEA1+ cells (Gargett, Schwab, and Deane 2016), Epithelial label retaining cells(Chan and Gargett 2006), CD44 + endometrial epithelial progenitors (Janzen et al. 2013) and ALDH1A1+ epithelial cells during development(Wu et al. 2017) implied that paracrine signals from the surrounding microenvironment were responsible for the mediation of hormonal regulation, a crosstalk between stem/progenitor cells with the surrounding niche cells was vital during the development, regeneration and might 
pathology(Gargett, Schwab, and Deane 2016; Wu et al. 2017; Chan and Gargett 2006; Janzen et al. 2013). Our atlas provided a dynamic connectivity map among subsets of the uterus.

TGF-beta family (TGF-beta, BMP), FGF, IGF1, EGF, PDGF, WNT were reported to regulate EMT through EMT transcription factors (SNAIL, TWIST, ZEB family), and inflammatory cytokines and hypoxia were shown to promote EMT by cross-talk with EMT TFs through STAT and HIF TF(Lamouille, Xu, and Derynck 2014)s. In the utrus, EMT was shown to be induced by estrogen through EMT TFs, and abnormal hormone level, dysregulation of WNT signaling would contribute to adenomyosis(Chen et al. 2010). Excessive ROS stress, abnormal exprsssion of ECM and ECM related LOX family, Lipocalin2 induced EMT is highly correlated with endometriosis through increased migratory and invasiveness properties(Vargha et al. 2008). Cytokines and chemokine, growth factors like TGFbeta signaling, hypoxia and oxidative stress are shown to be driver of EMT in endometrial cancer.(Bilyk et al. 2017)

\section{Conclusion:}

Here, we reconstructed an atlas of the human uterus tissues with the most detailed cell diversity, a compartmentalized cell ecosystem and a dynamic connectivity map of the human uterus tissue so far. Our atlas discovered new knowledge on uterus biology, would provide insight in the regeneration of uterus and reference for the pathogenesis of uterus.

\section{Materials and methods:}

\section{Human uterus collection}

The Human full-thickness uterus sample was collected from the normal portions of the uterus of leiomyoma patients from the First Affiliated Hospital, School of Medicine, Zhejiang University. Approval for utilizing the patient samples in this study was obtained from Ethics Committee of the First Affiliated Hospital, School of Medicine, Zhejiang University. 


\section{Single cell suspension preparation}

Single cell suspension was prepared according to previous study(Turco et al. 2017). Briefly, full-thickness uterus tissue was minced into small cubes with scissors, and digested with collagenase V (Sigma) in RPMI 1640 medium (Thermo Fisher Scientific) with gentle shaking every $20-30 \mathrm{~min}$ at $37^{\circ} \mathrm{C}$ for 2 hours. The stromal cells and smooth muscles were collected by passing the digested supernatant through $70 \mu \mathrm{m}$ cell sieves (Corning). The epithelial cell pellets were backwashed and further digested with TrypLE (Thermo Fisher Scientific) at $37^{\circ} \mathrm{C}$ for $10 \mathrm{~min}$. The digested supernatant was passed through $70 \mu \mathrm{m}$ cell sieves again to get single epithelial cell suspension. Finally, the stromal cells, smooth muscles and epithelial cells were combined to get the uterus single cell suspension for further analysis.

\section{Single cell capture, pre-amplification and sequencing}

Single cell capture, pre-amplification was conducted onto the GemCode instrument (10x Genomics) according to the manufactures' instructions (Chromium $^{\mathrm{TM}}$ Single Cell 3' Reagent Kit v2). The generated library was sequenced on five lanes of an Illumina X10 platform.

\section{Bioinformatic analysis}

The generated sequencing reads were aligned and analyzed using the Cell Ranger Pipeline (10x Genomics). We obtained 2735 cells with about 680k reads per cell with a median gene number per cell of 3,183. Single cell analysis was conducted using Seurat(Satija et al. 2015). Pseudo-space was reconstructed using Monocle (Trapnell et al. 2014). Connectivity map was constructed according to (Puram et al. 2017; Camp et al. 2017) using ligand-receptor dataset(Ramilowski et al. 2016). Gene ontology analysis was conducted using: http://geneontology.org.

\section{Histology and Immunostaining}

The human uterus tissue was fixed in $4 \%(\mathrm{w} / \mathrm{v})$ paraformaldehyde, and then dehydrated in an ethanol gradient, prior to embedment in paraffin and sectioning at $10 \mu \mathrm{m}$ thickness. Immunostaining were carried out as follows: The $10 \mu \mathrm{m}$ paraffin sections were rehydrated, antigen retrieved, rinsed three times with PBS, and treated 
with blocking solution (1\% BSA) for $30 \mathrm{~min}$, prior to incubation with primary antibodies at $4{ }^{\circ} \mathrm{C}$ overnight. The primary antibodies: rabbit anti-human antibodies against COL3A1 (Abcam, ab7778), ZEB1 (Proteintech Group, 21544-1-ap), KI67(Abcam, ab16667), NFkB (Cell Signaling TECHNOLOGY, 3987), $\gamma$ H2A.X(Cell Signaling TECHNOLOGY, 2577), mouse anti-human monoclonal antibodies against Pan-KRT (Abcam, ab7753), CD45(BD Biosciences, 555483), CD68(Abcam, ab955) were used to detect the expression of selected proteins within the human uterus. TUNEL assay kit was used to detect apoptotic cells (TEASEN, China) within the human uterus. Secondary antibody: goat anti-rabbit Alexa Fluor 488 (Invitrogen, A11008), donkey anti-mouse Alexa Fluor 488 (Invitrogen, A21202), goat anti-rabbit Alexa Fluor 546 (Invitrogen, A21430-f) and DAPI (Beyotime Institute of Biotechnology, China) were used to visualize the respective primary antibodies and the cell nuclei. All procedures were carried out according to the manufacturer's instructions.

\section{ACKNOWLEDGEMENTS:}

This work was supported by the National High Technology Research and Development Program of China (2017YFA0104902), the National Natural Science Foundation of China (CN) $(81270682,81300454)$, the Key Scientific and Technological Innovation Team of Zhejiang Province (2013TD11).

\section{AUTHOR CONTRIBUTIONS:}

B.W.: acquisition of clinical sample, data, data analysis and interpretation, manuscript writing; Y.L.: acquisition of sample, sample processing; Y.S.L.: processing of sample for immunostaining; K.X.J., K.Z., C.R.A., Q.K.L. data analysis; L.G.: manuscript preparation; W.Z., J.H.H., J.H.Q. acquisition of clinical sample; H.O.: conception and design, manuscript writing; X.Z.: conception and design, manuscript writing.

\section{DECLARATION OF INTERESTS:}

The authors declare no competing interests. 


\section{References:}

Bilyk, O., M. Coatham, M. Jewer, and L. M. Postovit. 2017. 'Epithelial-to-Mesenchymal Transition in the Female Reproductive Tract: From Normal Functioning to Disease Pathology', Front Oncol, 7: 145.

Cakmak, H., and H. S. Taylor. 2011. 'Implantation failure: molecular mechanisms and clinical treatment', Human Reproduction Update, 17: 242-53.

Camp, J. G., K. Sekine, T. Gerber, H. Loeffler-Wirth, H. Binder, M. Gac, S. Kanton, J. Kageyama, G. Damm, D. Seehofer, L. Belicova, M. Bickle, R. Barsacchi, R. Okuda, E. Yoshizawa, M. Kimura, H. Ayabe, H. Taniguchi, T. Takebe, and B. Treutlein. 2017. 'Multilineage communication regulates human liver bud development from pluripotency', Nature, 546: 533-+.

Chan, R. W., and C. E. Gargett. 2006. 'Identification of label-retaining cells in mouse endometrium', Stem Cells, 24: 1529-38.

Chen, Y. J., H. Y. Li, C. H. Huang, N. F. Twu, M. S. Yen, P. H. Wang, T. Y. Chou, Y. N. Liu, K. C. Chao, and M. H. Yang. 2010. 'Oestrogen-induced epithelial-mesenchymal transition of endometrial epithelial cells contributes to the development of adenomyosis', Journal of Pathology, 222: 261-70.

Cousins, F. L., P. M. Kirkwood, P. T. K. Saunders, and D. A. Gibson. 2016. 'Evidence for a dynamic role for mononuclear phagocytes during endometrial repair and remodelling', Scientific Reports, 6.

Diaz-Gimeno, P., M. Ruiz-Alonso, D. Blesa, and C. Simon. 2014. 'Transcriptomics of the human endometrium', International Journal of Developmental Biology, 58: 127-37.

Evans, G. E., J. A. Martinez-Conejero, G. T. M. Phillipson, P. H. Sykes, I. L. Sin, E. Y. N. Lam, C. G. Print, J. A. Horcajadas, and J. J. Evans. 2014. 'In the secretory endometria of women, luminal epithelia exhibit gene and protein expressions that differ from those of glandular epithelia', Fertility and Sterility, 102: 307-+.

Fu, B. Q., Y. G. Zhou, X. Ni, X. H. Tong, X. X. Xu, Z. J. Dong, R. Sun, Z. G. Tian, and H. M. Wei. 2017. 'Natural Killer Cells Promote Fetal Development through the Secretion of Growth-Promoting Factors', Immunity, 47: 1100-+. 
Gargett, C. E. 2007. 'Uterine stem cells: what is the evidence?', Hum Reprod Update, 13: $87-101$.

Gargett, C. E., H. P. T. Nguyen, and L. Ye. 2012. 'Endometrial regeneration and endometrial stem/progenitor cells', Reviews in Endocrine \& Metabolic Disorders, 13: 235-51.

Gargett, C. E., K. E. Schwab, and J. A. Deane. 2016. 'Endometrial stem/progenitor cells: the first 10 years', Hum Reprod Update, 22: 137-63.

Hufnagel, D., F. Li, E. Cosar, G. Krikun, and H. S. Taylor. 2015. 'The Role of Stem Cells in the Etiology and Pathophysiology of Endometriosis', Seminars in Reproductive Medicine, 33: 333-40.

Ibrahim, M. G., M. Sillem, J. Plendl, V. Chiantera, J. Sehouli, and S. Mechsner. 2017. 'Myofibroblasts Are Evidence of Chronic Tissue Microtrauma at the Endometrial-Myometrial Junctional Zone in Uteri With Adenomyosis', Reproductive Sciences, 24: 1410-18.

Janzen, D. M., D. Cheng, A. M. Schafenacker, D. Y. Paik, A. S. Goldstein, O. N. Witte, A. Jaroszewicz, M. Pellegrini, and S. Memarzadeh. 2013. 'Estrogen and progesterone together expand murine endometrial epithelial progenitor cells', Stem Cells, 31: 808-22.

Krjutskov, K., S. Katayama, M. Saare, M. Vera-Rodriguez, D. Lubenets, K. Samuel, T. Laisk-Podar, H. Teder, E. Einarsdottir, A. Salumets, and J. Kere. 2016. 'Single-cell transcriptome analysis of endometrial tissue', Human Reproduction, 31: 844-53.

Lamouille, S., J. Xu, and R. Derynck. 2014. 'Molecular mechanisms of epithelial-mesenchymal transition', Nat Rev Mol Cell Biol, 15: 178-96.

Liu, X. S., M. H. Shen, Q. M. Qi, H. Q. Zhang, and S. W. Guo. 2016. 'Corroborating evidence for platelet-induced epithelial-mesenchymal transition and fibroblast-to-myofibroblast transdifferentiation in the development of adenomyosis', Human Reproduction, 31: 734-49.

Maybin, J. A., and H. O. D. Critchley. 2015. 'Menstrual physiology: implications for endometrial pathology and beyond', Human Reproduction Update, 21: 748-61. 
Merrell, A. J., and B. Z. Stanger. 2016. 'Adult cell plasticity in vivo: de-differentiation and transdifferentiation are back in style', Nat Rev Mol Cell Biol, 17: 413-25.

Nguyen, H. P. T., C. N. Sprung, and C. E. Gargett. 2012. 'Differential Expression of Wnt Signaling Molecules Between Pre- and Postmenopausal Endometrial Epithelial Cells Suggests a Population of Putative Epithelial Stem/Progenitor Cells Reside in the Basalis Layer', Endocrinology, 153: 2870-83.

Ono, M., T. Maruyama, H. Masuda, T. Kajitani, T. Nagashima, T. Arase, M. Ito, K. Ohta, H. Uchida, H. Asada, Y. Yoshimura, H. Okano, and Y. Matsuzaki. 2007. 'Side population in human uterine myometrium displays phenotypic and functional characteristics of myometrial stem cells', Proceedings of the National Academy of Sciences of the United States of America, 104: 18700-05.

Paria, B. C., X. Zhao, S. K. Das, S. K. Dey, and K. Yoshinaga. 1999. 'Zonula occludens-1 and E-cadherin are coordinately expressed in the mouse uterus with the initiation of implantation and decidualization', Dev Biol, 208: $488-501$.

Patterson, A. L., L. Zhang, N. A. Arango, J. Teixeira, and J. K. Pru. 2013. 'Mesenchymal-to-epithelial transition contributes to endometrial regeneration following natural and artificial decidualization', Stem Cells Dev, 22: 964-74.

Proserpio, V., and T. Lonnberg. 2016. 'Single-cell technologies are revolutionizing the approach to rare cells', Immunology and Cell Biology, 94: 225-29.

Puram, S. V., I. Tirosh, A. S. Parikh, A. P. Patel, K. Yizhak, S. Gillespie, C. Rodman, C. L. Luo, E. A. Mroz, K. S. Emerick, D. G. Deschler, M. A. Varvares, R. Mylvaganam, O. Rozenblatt-Rosen, J. W. Rocco, W. C. Faquin, D. T. Lin, A. Regev, and B. E. Bernstein. 2017. 'Single-Cell Transcriptomic Analysis of Primary and Metastatic Tumor Ecosystems in Head and Neck Cancer', Cell, 171: 1611-+.

Ramilowski, J. A., T. Goldberg, J. Harshbarger, E. Kloppmann, M. Lizio, V. P. Satagopam, M. Itoh, H. Kawaji, P. Carninci, B. Rost, and A. R. R. Forrest. 2016. 'A draft network of ligand-receptor-mediated multicellular signalling in human (vol 6, 7866, 2015)', Nature Communications, 7. 
Satija, R., J. A. Farrell, D. Gennert, A. F. Schier, and A. Regev. 2015. 'Spatial reconstruction of single-cell gene expression data', Nature Biotechnology, 33: 495-U206.

Seli, E., and A. Arici. 2003. 'Endometriosis: Interaction of immune and endocrine systems', Seminars in Reproductive Medicine, 21: 135-44.

Tetteh, P. W., O. Basak, H. F. Farin, K. Wiebrands, K. Kretzschmar, H. Begthel, M. van den Born, J. Korving, F. de Sauvage, J. H. van Es, A. van Oudenaarden, and H. Clevers. 2016. 'Replacement of Lost Lgr5-Positive Stem Cells through Plasticity of Their Enterocyte-Lineage Daughters', Cell Stem Cell, 18: 203-13.

Trapnell, C., D. Cacchiarelli, J. Grimsby, P. Pokharel, S. Q. Li, M. Morse, N. J. Lennon, K. J. Livak, T. S. Mikkelsen, and J. L. Rinn. 2014. 'The dynamics and regulators of cell fate decisions are revealed by pseudotemporal ordering of single cells', Nature Biotechnology, 32: 381-U251.

Tritschler, S., F. J. Theis, H. Licked, and A. Bottcher. 2017. 'Systematic single-cell analysis provides new insights into heterogeneity and plasticity of the pancreas', Molecular Metabolism, 6: 974-90.

Turco, M. Y., L. Gardner, J. Hughes, T. Cindrova-Davies, M. J. Gomez, L. Farrell, M. Hollinshead, S. G. E. Marsh, J. J. Brosens, H. O. Critchley, B. D. Simons, M. Hemberger, B. K. Koo, A. Moffett, and G. J. Burton. 2017. 'Long-term, hormone-responsive organoid cultures of human endometrium in a chemically defined medium', Nature Cell Biology, 19: 568-+.

Valentijn, A. J., K. Palial, H. Al-Lamee, N. Tempest, J. Drury, T. Von Zglinicki, G. Saretzki, P. Murray, C. E. Gargett, and D. K. Hapangama. 2013. 'SSEA-1 isolates human endometrial basal glandular epithelial cells: phenotypic and functional characterization and implications in the pathogenesis of endometriosis', Hum Reprod, 28: 2695-708.

van Kaam, K. J. A. F., J. P. Schouten, A. W. Nap, G. A. J. Dunselman, and P. G. Groothuis. 2008. 'Fibromuscular differentiation in deeply infiltrating endometriosis is a reaction of resident fibroblasts to the presence of ectopic endometrium', Human Reproduction, 23: 2692-700. 
Varga, J., and F. R. Greten. 2017. 'Cell plasticity in epithelial homeostasis and tumorigenesis', Nat Cell Biol, 19: 1133-41.

Vargha, R., T. O. Bender, A. Riesenhuber, M. Endemann, K. Kratochwill, and C. Aufricht. 2008. 'Effects of epithelial-to-mesenchymal transition on acute stress response in human peritoneal mesothelial cells', Nephrol Dial Transplant, 23: 3494-500.

Wu, B., C. An, Y. Li, Z. Yin, L. Gong, Z. Li, Y. Liu, B. C. Heng, D. Zhang, H. Ouyang, and X. Zou. 2017. 'Reconstructing Lineage Hierarchies of Mouse Uterus Epithelial Development Using Single-Cell Analysis', Stem Cell Reports, 9: $381-96$.

Zepp, J. A., W. J. Zacharias, D. B. Frank, C. A. Cavanaugh, S. Zhou, M. P. Morley, and E. E. Morrisey. 2017. 'Distinct Mesenchymal Lineages and Niches Promote Epithelial Self-Renewal and Myofibrogenesis in the Lung', Cell, 170: 1134-48. 


\section{Figure legend:}

Figure 1 Single cell RNA-seq of full-thickness human uterus. (A) Workflow shows sample processing, enzymatic digestion and drop-seq based single cell RNA-seq. (B) t-distributed stochastic neighbor embedding (t-SNE) plot of 2735 single cell from a full-thickness secretory phase uterus by single cell RNA-seq (scRNA-seq). (C) Heatmap shows differential expressed gene signatures of each cell cluster from scRNA-seq. (D) t-SNE plot of selected marker genes from gene signatures of each cell cluster (KRT18, KRT8, EPCAM, CLDN3 for epithelial cell; MME, FN1, COL3A1, HOXA10 for stroma cells; ACTA2, MYH11, MYL6, MYL9 for SMA+ cells (smooth muscle cells and myofibroblasts); CD34, VWF, CLDN5, SOX18 for endothelial cells; PTPRC, CD68, CD163, CD96 for immune cells). (E)Each cell cluster (epithelial cell, stroma cell, endothelial cell, SMA+ cell, immune cells) was further clustered into their relevant subpopulations (32 sub-clusters in total).

Figure 2 Heterogeneity of uterus epithelia subsets. (A) t-distributed stochastic neighbor embedding (t-SNE) plot of epithelial cells using Seurat. (B) Heatmap shows differential expressed gene signature of each sub-cluster from epithelial cells. (C) Violin plot depict markers (TUBA1A and TUBA1B) of ciliated epithelial sub-clusters (Epi4, Epi5, Epi6, Epi9, Epi10). (D) Violin plot depict markers of secretory epithelial sub-clusters (Epi0, Epi1, Epi2, Epi3, Epi7, Epi8, Epi9) as labelled by secretoglobin family (SCGB1D4, SCGB2A1) and inflammatory cytokines and chemokines (CXCL, VEGFA). (E) Violin plot depict markers (KRT8, COL3A1) and transcription factors (ZEB1, SNAI2) of EMT epithelial sub-cluster(Epi10). (F) Immunofluorescent staining showed the expression of EMT markers (pan-KRT, COL3A1, ZEB1) in the upper layer of the functionalis of the endometria. (G) Gene ontology analysis of the highly expressed genes in the EMT sub-cluster (Epi10). (H) Pseudospace ordering of all the epithelial subclusters.

Figure 3 Heterogeneity of uterus stroma subsets. (A) t-SNE plot of stromal cells using Seurat. (B) Heatmap shows differential expressed gene signature of each sub-cluster from stromal cells. (C) Gene ontology analysis of the highly expressed genes in the stromal sub-cluster Stro0. (D) Gene ontology analysis of the highly 
expressed genes in the stromal sub-cluster Stro2. (E) Gene ontology analysis of the highly expressed genes in the stromal sub-cluster Stro3. (F) Gene ontology analysis of the highly expressed genes in the stromal sub-cluster Stro4. (G) Gene ontology analysis of the highly expressed genes in the stromal sub-cluster Stro5.(H) Pseudospace ordering of all the stromal sub-clusters.

Figure 4 Heterogeneity of uterus endothelial subsets. (A) t-SNE plot of endothelial cells using Seurat. (B) Heatmap shows differential expressed gene signature of each sub-cluster from endothelial cells. (C) Violin plot depict selected markers of each endothelial sub-cluster. (D) Gene ontology analysis of the highly expressed genes in the endothelial sub-cluster Endo0. (E) Gene ontology analysis of the highly expressed genes in the endothelial sub-cluster Endo1. (F) Gene ontology analysis of the highly expressed genes in the endothelial sub-cluster Endo2. (G) Gene ontology analysis of the highly expressed genes in the endothelial sub-cluster Endo4. (H) Pseudospace ordering of all the endothelial sub-clusters.

Figure 5 Heterogeneity of uterus smooth muscle cell and myofibroblast subsets. (A) t-SNE plot of SMA+ cells using Seurat. (B) Heatmap shows differential expressed gene signature of each sub-cluster from SMA+ cells. (C) Violin plot depict selected markers of smooth muscle cell (SMA1, SMA3) markers (ACTG2, ACTA2, DES, MYH11) and myofibroblast (SMA0, SMA4) markers (COL4A1, ACTA2). (D) Gene ontology analysis of the highly expressed genes in the myofibroblast SMA0. (E) Gene ontology analysis of the highly expressed genes in the myometrial cell SMA1. (F) Gene ontology analysis of the highly expressed genes in the sub-cluster SMA2. (G) Gene ontology analysis of the highly expressed genes in the myofibroblast SMA4. (H) Gene ontology analysis of the highly expressed genes in the vascular smooth muscle cell SMA3.

Figure 6 Heterogeneity of uterus immune cell subsets. (A) t-SNE plot of immune cells depict sun-clusters of macrophage and NK cells. (B) Violin plot depict selected markers of macrophage(CD86,CD163) and NK cells(CD96). Heatmaps show Top20 differential expressed gene signature of each sub-cluster from macrophage(C) and NK cells(D). (E) Violin plot depict selected functional markers (C1QB, CSF1R, TLR4, 
IL1B) of macrophage and NK cells.

Figure 7 Stress, inflammatory and apoptotic subsets in the uterus ecosystem. Immunofluorescent staining showed the representative number of CD45 positive immune cells (A, green), CD68 macrophages (B, green), TUNEL positive apoptotic cells (C \& E, red), NFkB inflammatory state cells (C, green), proliferative cells (D, red) and DNA damage $\gamma \mathrm{H} 2 \mathrm{~A} . \mathrm{X}+$ cells $(\mathrm{E}$, green) in the secretory full-thickness human uterus (three layers: functionalis, basalis layer and myometrium). All the nucleus was stained with DAPI(blue). Scale bar,50 $\mu \mathrm{m}$.

Figure 8 Connectivity map of human uterus subsets. (A) Heatmap shows the total numbers of putative receptor-ligand interactions between two sub-clusters from the ecosystem of the full-thickness secretory uterus. (B) Circle plot depicts the total number of ligand-receptor interactions between each sub-cluster from the EMT microenvironment and the EMT sub-cluster (epi10). (C) Heatmap shows the top ligand-receptor interactions between each sub-cluster from the EMT microenvironment and the EMT sub-cluster. (D) Gene ontology analysis of the ligands from the top ligand-receptor interactions between each sub-cluster from the EMT microenvironment and the EMT sub-cluster. 


\section{Figure1:}

A

\section{C}

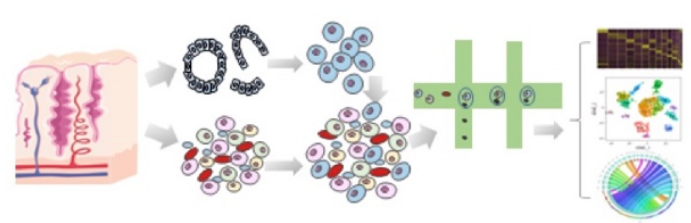

B

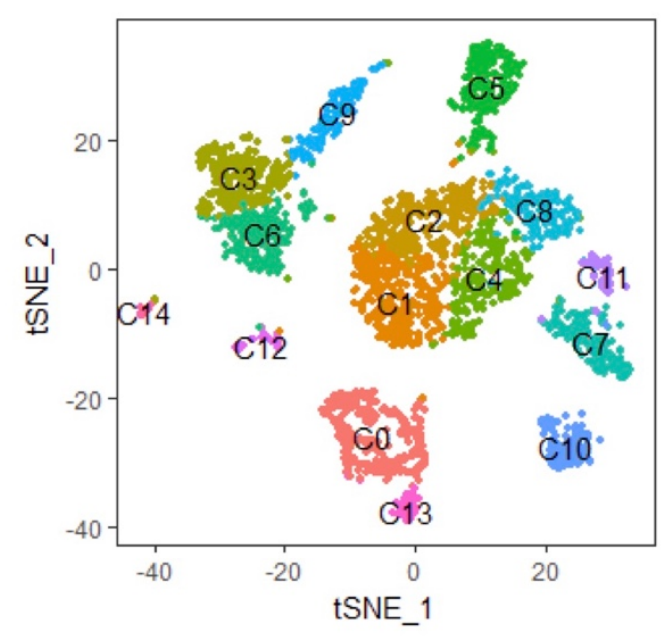

D
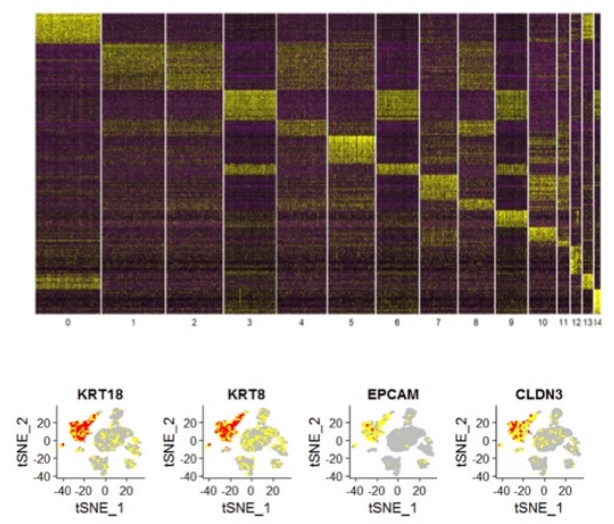

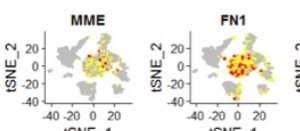

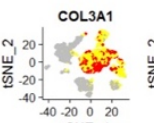

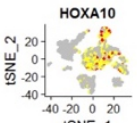

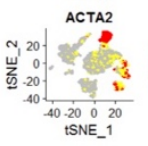

20. MYH11

紫

$\underset{40.200}{200}$

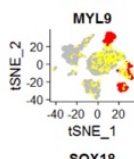

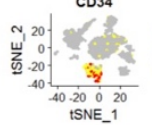

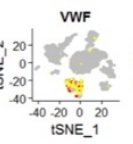

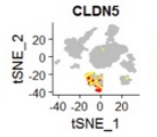

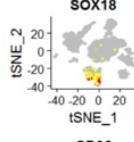

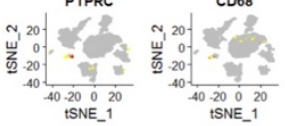

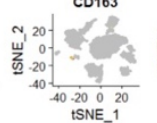

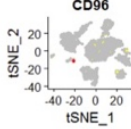

$\mathrm{E}$

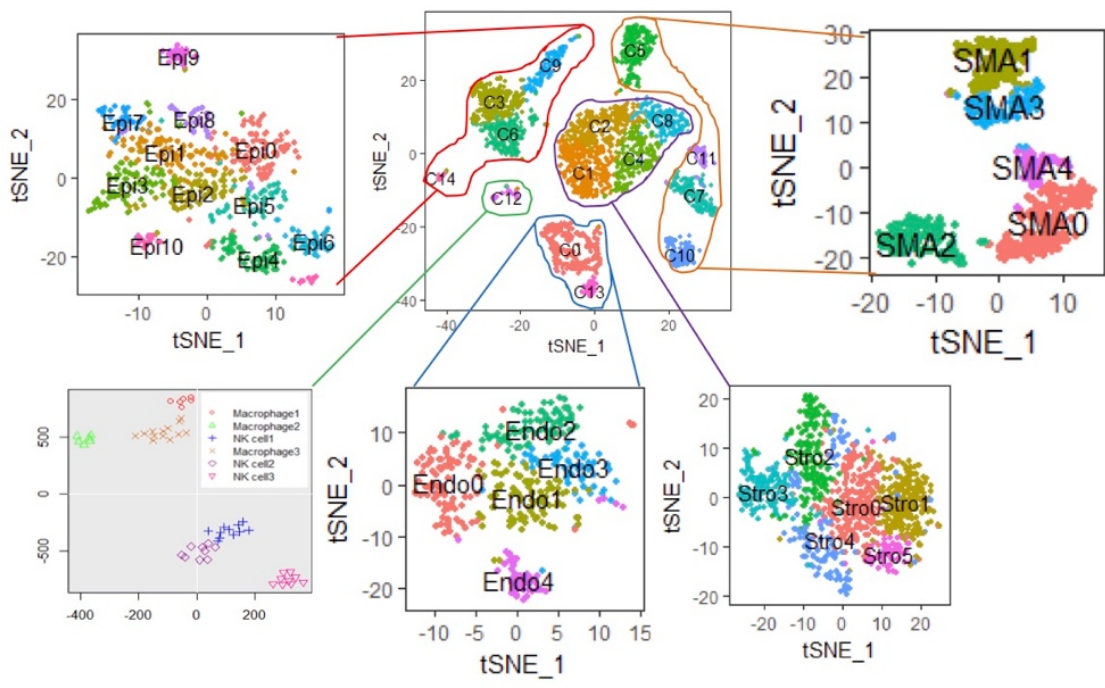




\section{Figure2:}

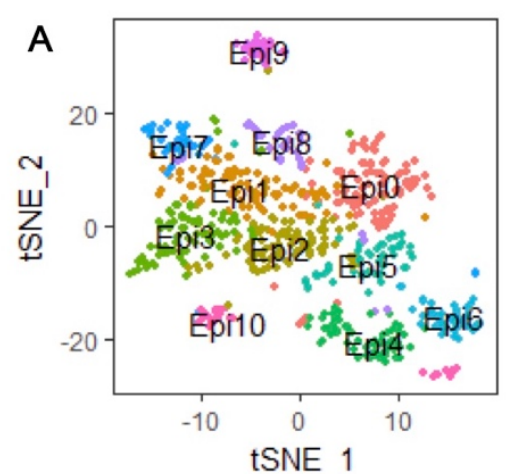

C tubA1A TUBA1B
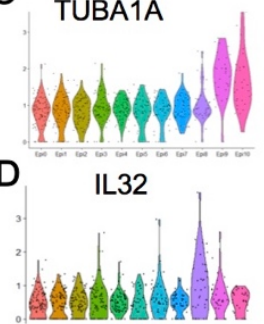

CXCL8

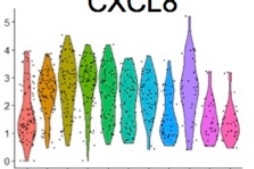

SCGB1D2
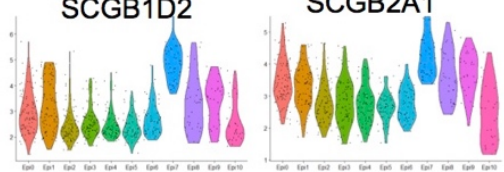

$\mathrm{E}$

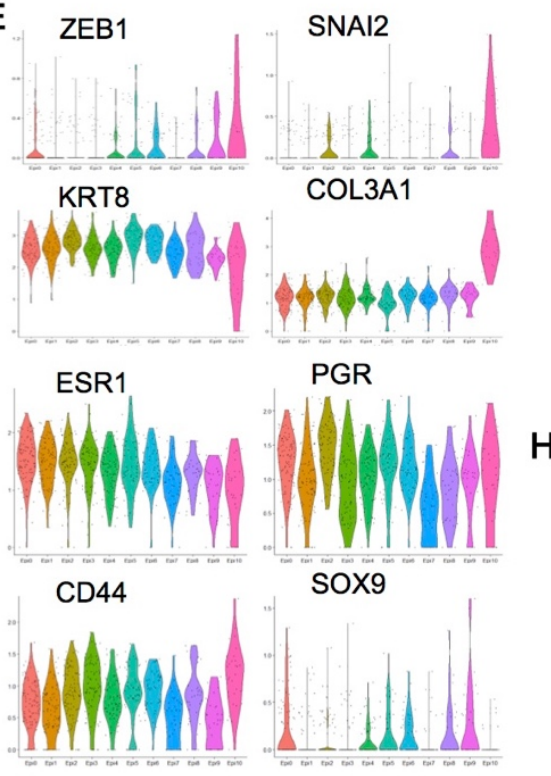

G
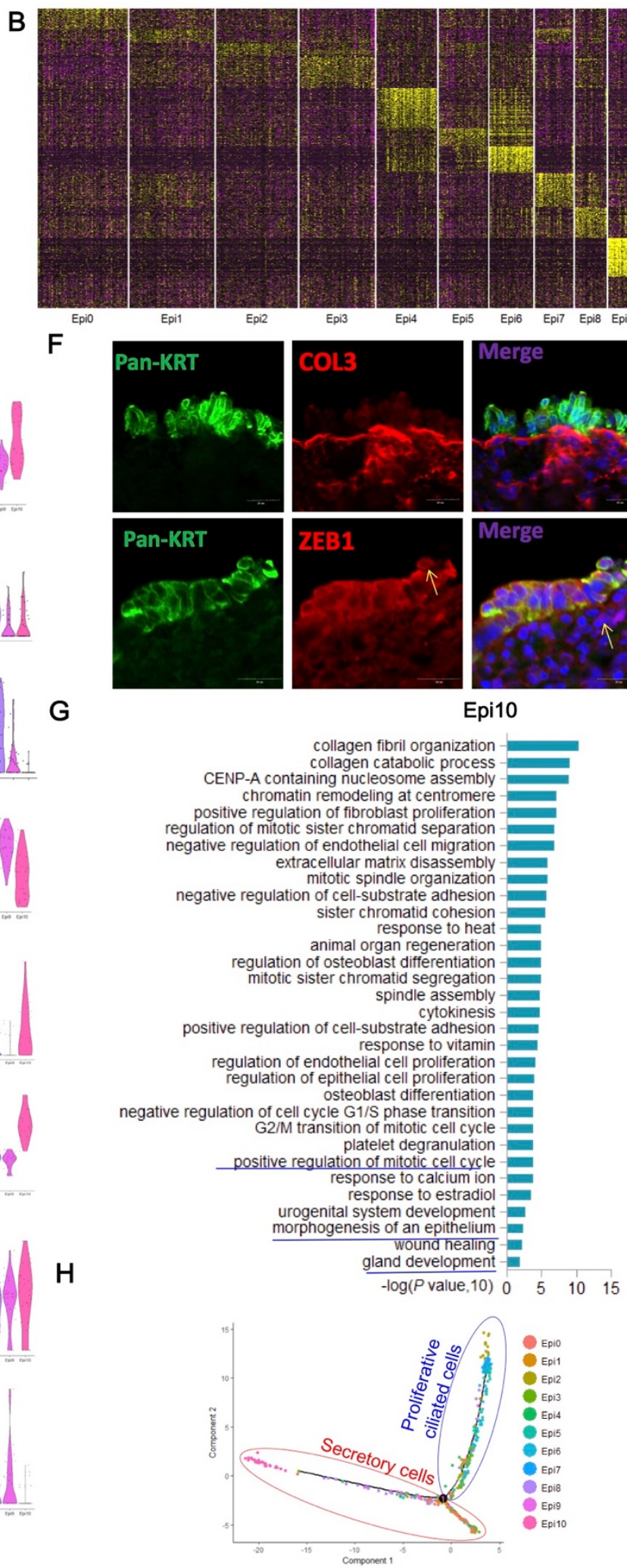
bioRxiv preprint doi: https://doi.org/10.1101/267849; this version posted February 19, 2018. The copyright holder for this preprint (which

was not certified by peer review) is the author/funder. All rights reserved. No reuse allowed without permission.

\section{Figure3:}
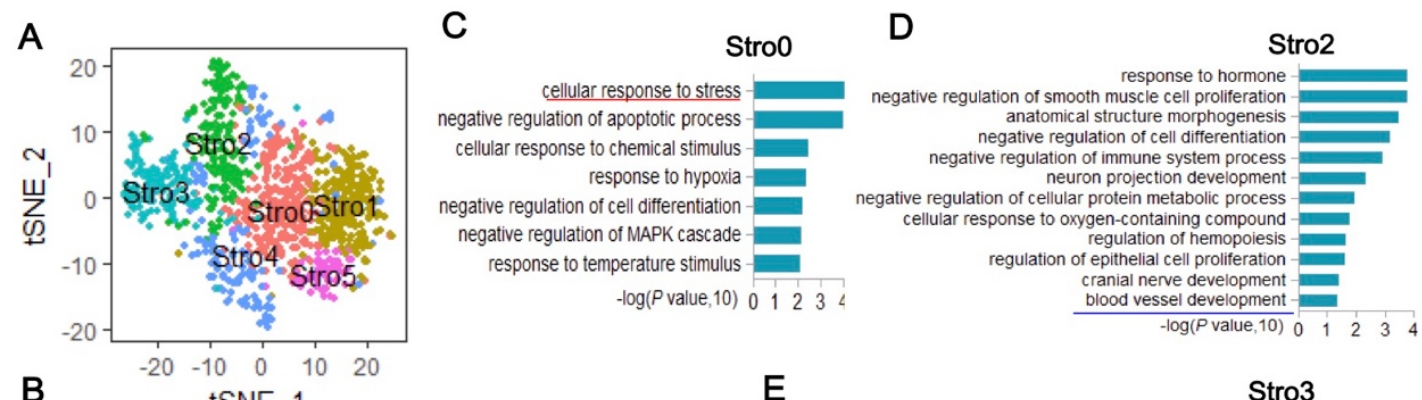

tSNE 1

E

Stro3

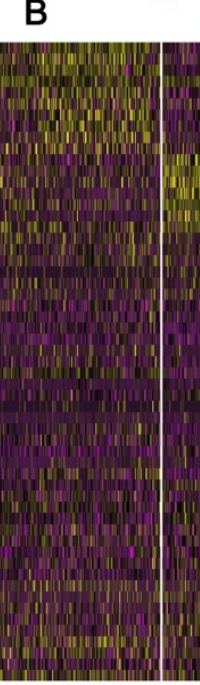

F
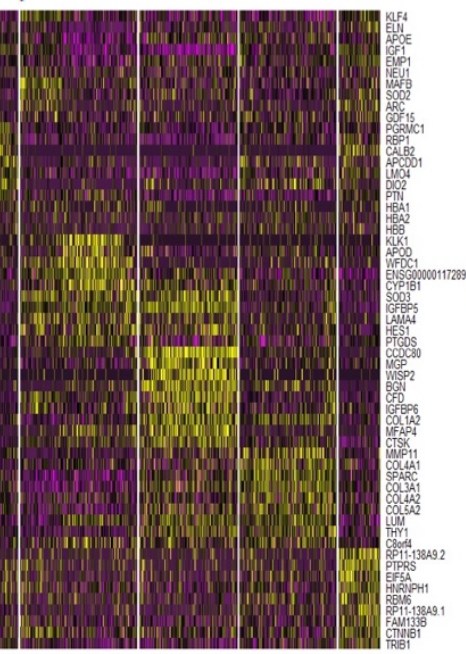

Stro4

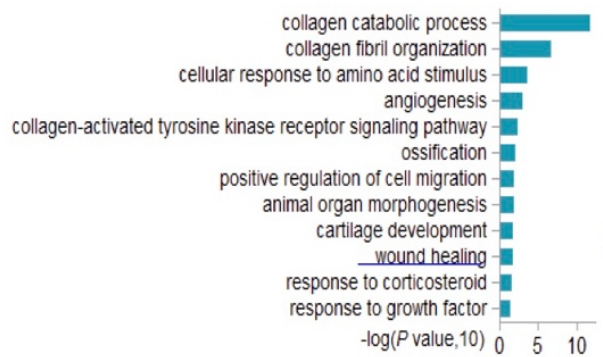

$\mathrm{H}$

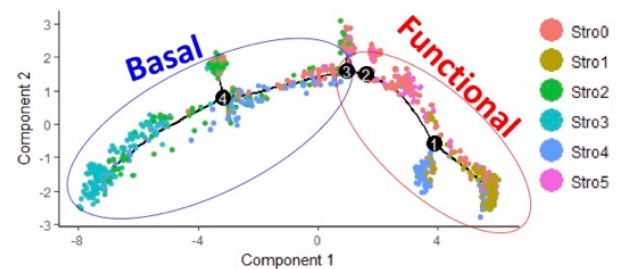

cell adhesion

regulation of cell growth

collagen catabolic process

skeletal system development

collagen fibril organization

positive regulation of cell proliferation

platelet degranulation
response to acid chemical

anatomical structure formation involved in morphogenesisis

regulation of endopeptidase activity

negative reguation of immune system process
negative regulation of cellular response to growth factor stimulus

gland development
response to hormone

positive regulation of cell differentiation

blood vessel mourphogeanesis

negative regulation of smooth muscle cell proliferation

post-translational protein modification -

muscle structure development issue morphogenesis -

regulation of TGFbeta receptor signaling patthway

keratan suffate catabolic process -

positive regulation of IGF receptor signaling pathway -

$\begin{array}{llll}-\log (P \text { value }, 10) & 0 & 5 & 10\end{array}$

G

\section{Stro5}

negative regulation of apoptotic process
positive regulation of epithelial cell proliferation regulation of biological quality ion of developmental process circulatory system development regulation of angiogenesis anatomical structure morphogenesis

negative regulation of cellular component organization -
dendritic spine organization positive regulation of cyclin-dependent protein serine/threonine kinase activity regulation organ morphogenesis

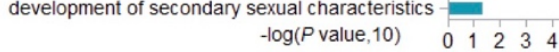

$$
\text { log( Palue, } 10)
$$


bioRxiv preprint doi: https://doi.org/10.1101/267849; this version posted February 19, 2018. The copyright holder for this preprint (which

was not certified by peer review) is the author/funder. All rights reserved. No reuse allowed without permission.

\section{Figure4:}

A

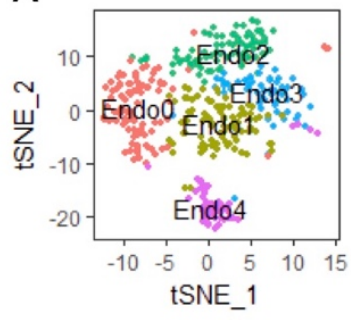

B

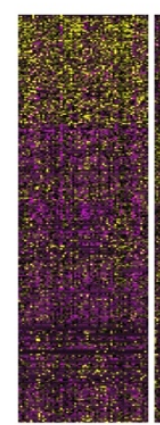

$\mathrm{O}$

C

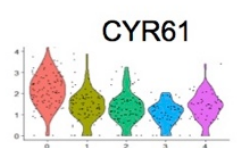

1

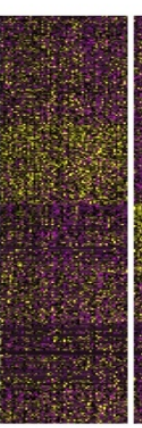

2
D
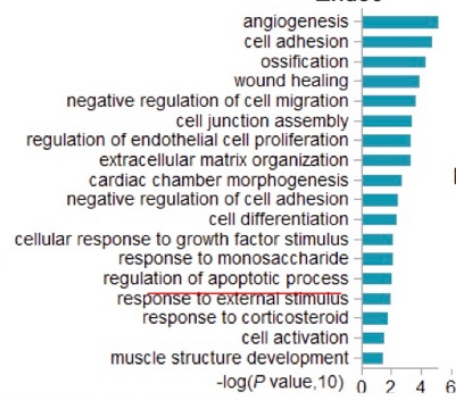

$-\log (P$ value, 10) $0 \quad 2 \quad 4 \quad 4 \quad 6$

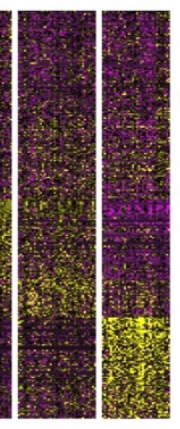

34
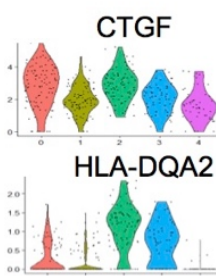

LIFR

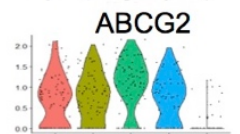

CXCL12

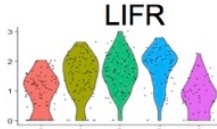

CLDN5

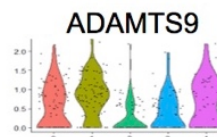

CXCL2

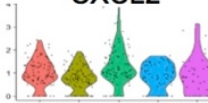

ADAMTS6

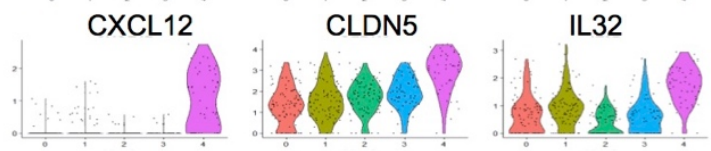

$\mathrm{H}$

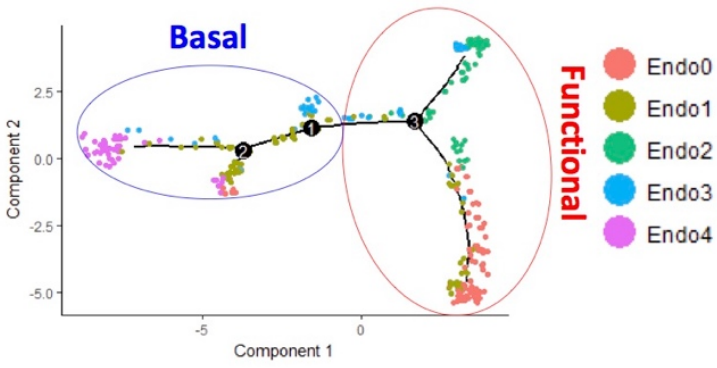

E

Endo1

regulation of phosphorylation regulation of response to stimulus regulation of epithelial cell migration blood vessel development. regulation of immune system process positive regulation of phosphate metabolic process morphogenesis of an epithelium positive regulation of cell death positive regulation of cell motility regulation of phospholipid metabolic process -

negative regulation of cell proliferation $-\log \left(P\right.$ value, 10) $0 \begin{array}{lllll}0 & 1 & 2 & 3 & 4\end{array}$

$\mathbf{F}$

\section{Endo2}

antigen processing and presentation of exogenous peptide antigen via MHC class I interferon-gamma-mediated signaling pathway T cell costimulation peptide antigen assembly with MHC class $\|$ protein complex T cell receptor signaling pathway -

G $-\log (P$ value, 10$) \quad 0 \quad 2 \quad 4 \quad 6$

Endo4

angiogenesis differentiation cell adhesion

cellular response to TGF beta stimulus cell morphogenesis involved in differentiation animal organ morphogenesis endothelial cell development extracellular matrix organization axon development positive regulation of cell differentiation regulation of phosphate metabolic process kidney development regulation of stress fiber assembly cell-cell junction assembly positive regulation of chemotaxis cell surface receptor signaling pathway $-\log (P$ value, 10$) 02468$ 
bioRxiv preprint doi: https://doi.org/10.1101/267849; this version posted February 19, 2018. The copyright holder for this preprint (which

was not certified by peer review) is the author/funder. All rights reserved. No reuse allowed without permission.

\section{Figure5:}

A

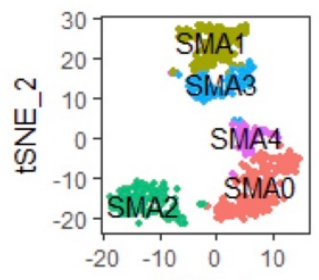

tSNE_1
B

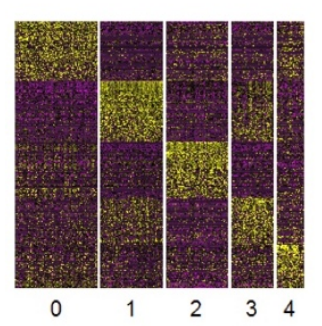

C

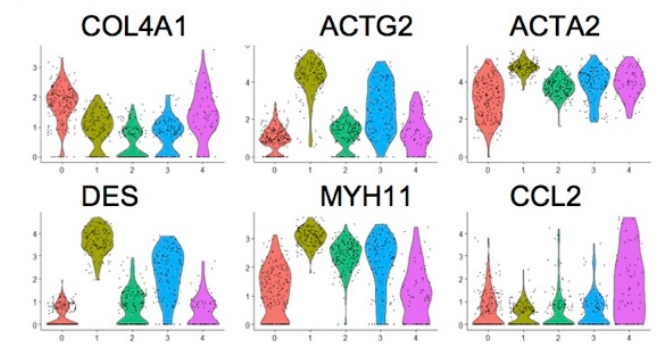

E myofibroblast -SMAO

negative regulation of cell proliferation angiogenesis animal organ morphogenesis Notch signaling pathway regulation of cell migration positive regulation of hydrolase activity cell-substrate adhesion central nervous system development collagen fibril organization aorta morphogenesis tube development response to hypoxia glomerulus development response to oxidative stress heart valve development regulation of apoptotic process

$$
-\log (P \text { value, } 10) \quad 02246810
$$

F

SMA2

cellular response to zinc ion regeneration

regulation of muscle system process

mRNA catabolic process nonsense-mediated decay SRP-dependent cotranslational protein targeting to membrane muscle system process negative regulation of growth $-\log (P$ value, 10$) 0 \begin{array}{lllll}0 & 2 & 3 & 4 & 5\end{array}$ vascular smooth muscle -SMA3

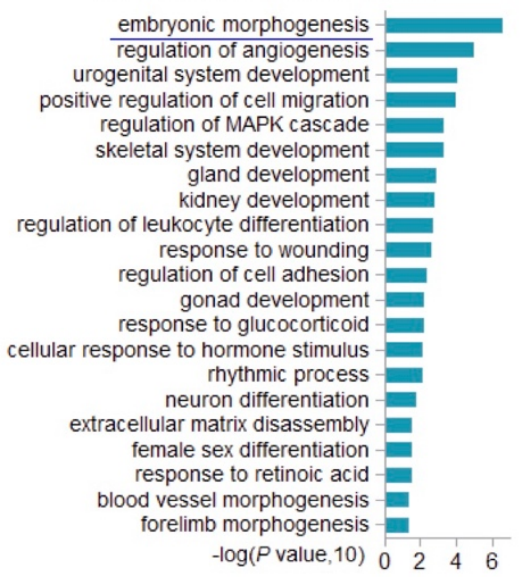
positive regulation of supramolecular fiber organization extracellular matrix organization positive regulation of cytoskeleton organization cell junction assembly actomyosin structure organization muscle organ development positive regulation of cell migration actin filament fragmentation regulation of actin filament organization smooth muscle contraction cell-matrix adhesion

positive regulation of sodium ion transporter activity striated muscle cell development circulatory system development regulation of muscle contraction morphogenesis of an epithelium actin-mediated cell contraction

G $\begin{array}{lllllll}-\log (P \text { value }, 10) & 0 & 1 & 2 & 3 & 4 & 5\end{array}$

myofibroblast -SMA4

negative regulation of cell differentiation negative regulation of cell migration negative regulation of cell proliferation response to interferon-gamma negative regulation of immune system process type I interferon signaling pathway urogenital system development gulation of osteoblast differentiation regulation of cell-substrate adhesion cellular response to growth factor stimulus negative regulation of apoptotic process collagen fibril organization angiogenesis regulation of endothelial cell proliferation wound healing leukocyte migration regulation of peptidase activity enzyme linked receptor protein signaling pathway - $\log (P$ value, 10 $) \quad 0 \quad 2 \quad 4 \quad 6$ 


\section{Figure6:}

A

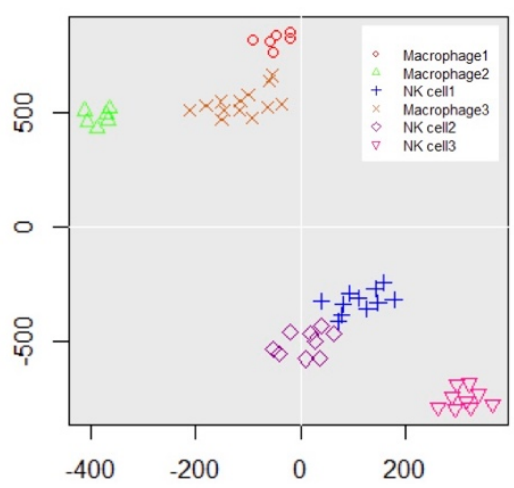

C

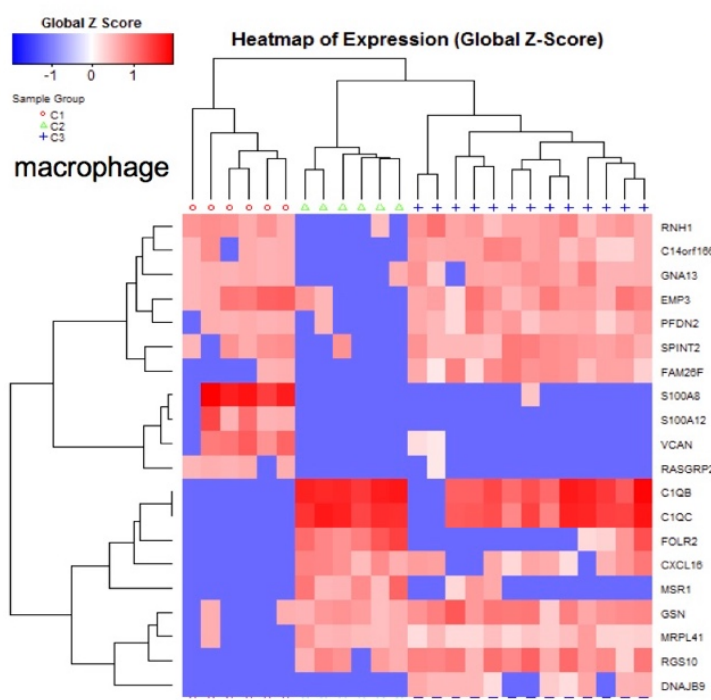

E

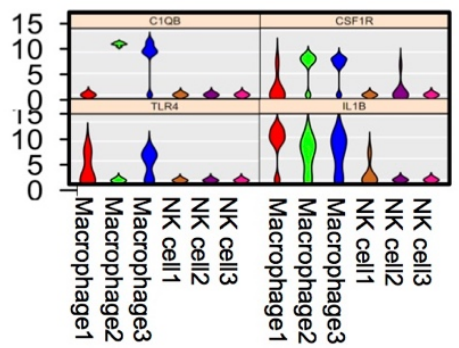

B

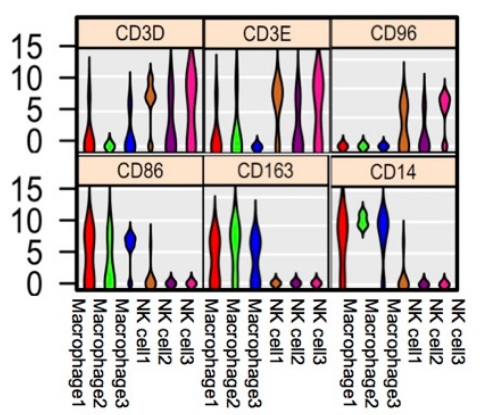

D

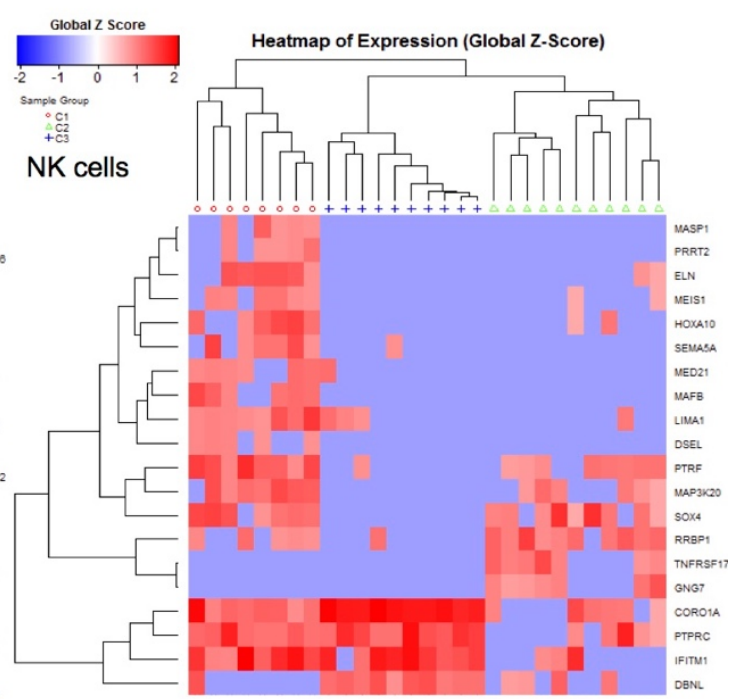




\section{Figure7:}

A

Functionalis

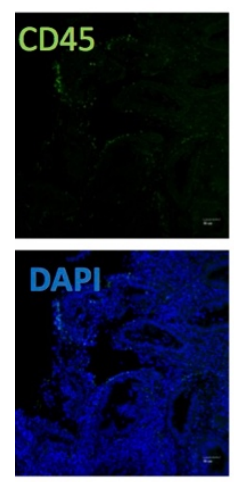

C

C Functionalis

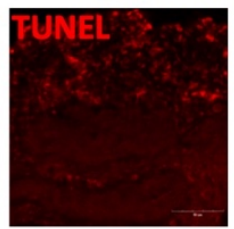

NFkB
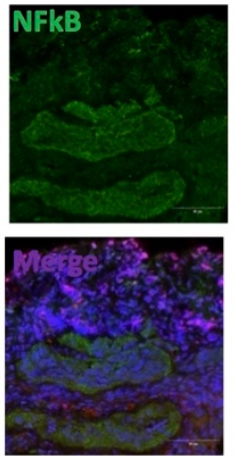

E Functionalis
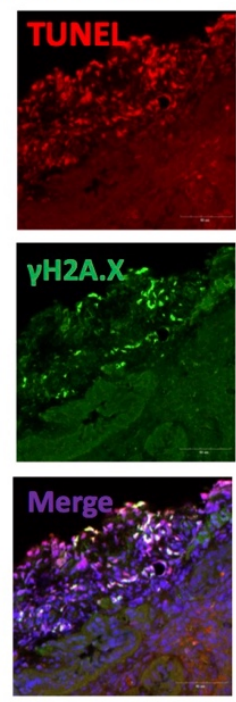

B

\section{Myometrium Functionalis}
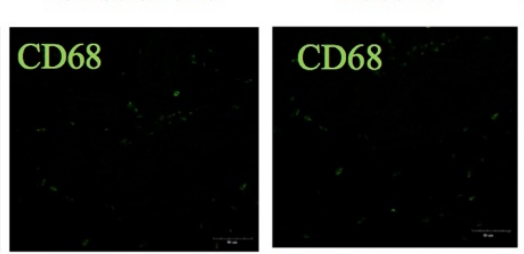

Myometrium
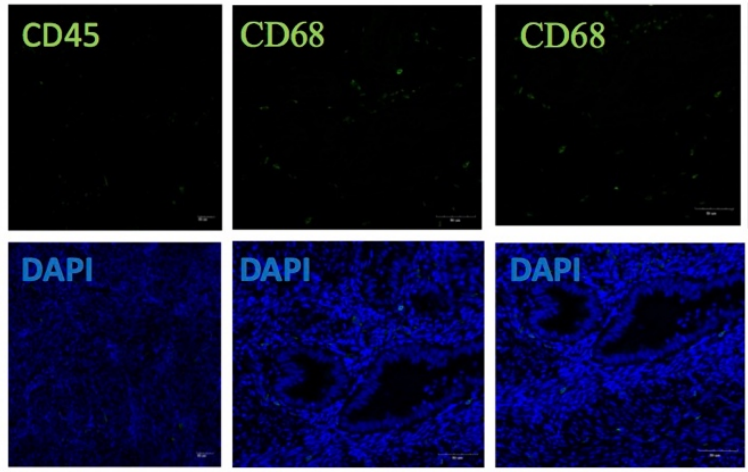

D
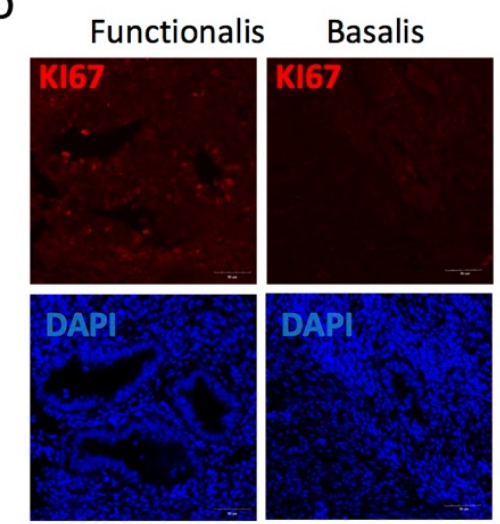

Myometrium
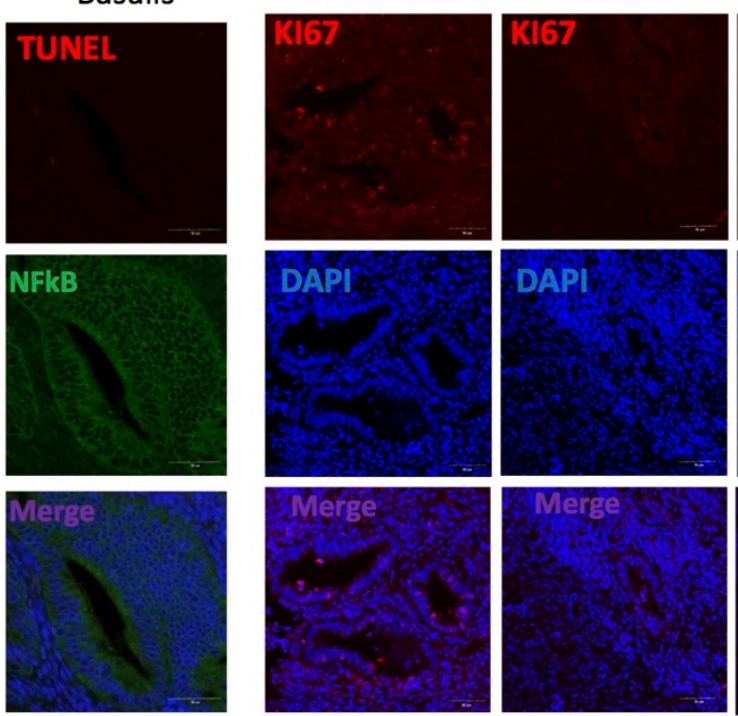

Myometrium

Functionalis

Basalis
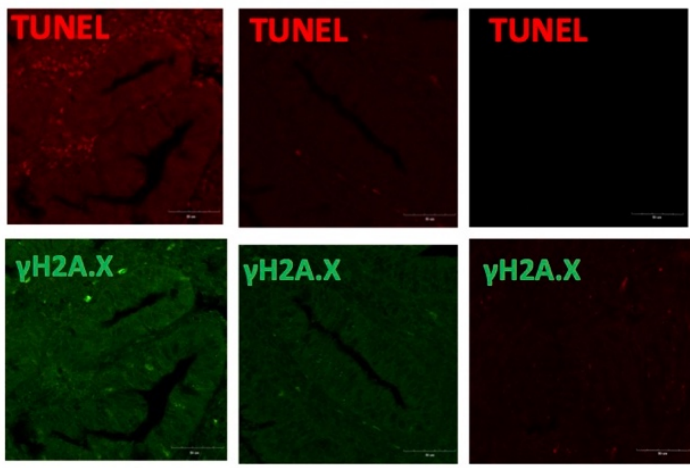

YH2A.X
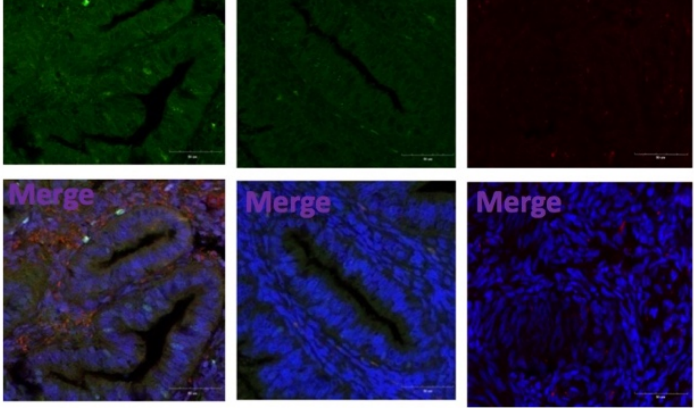


\section{Figure8:}

A

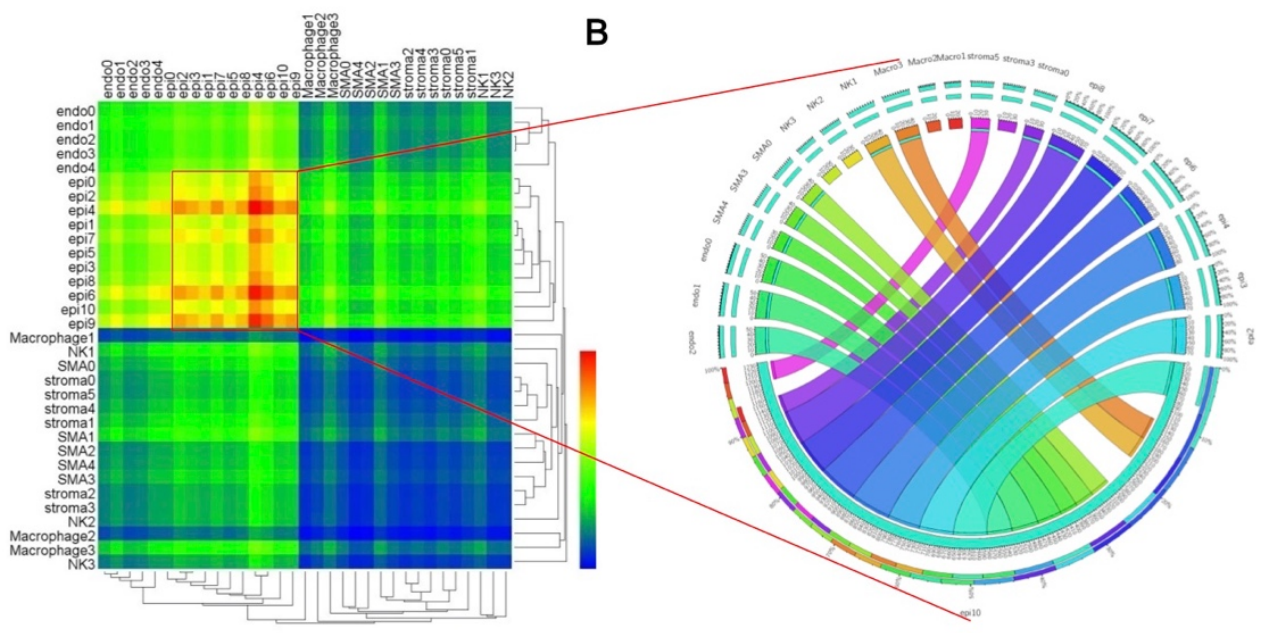

C

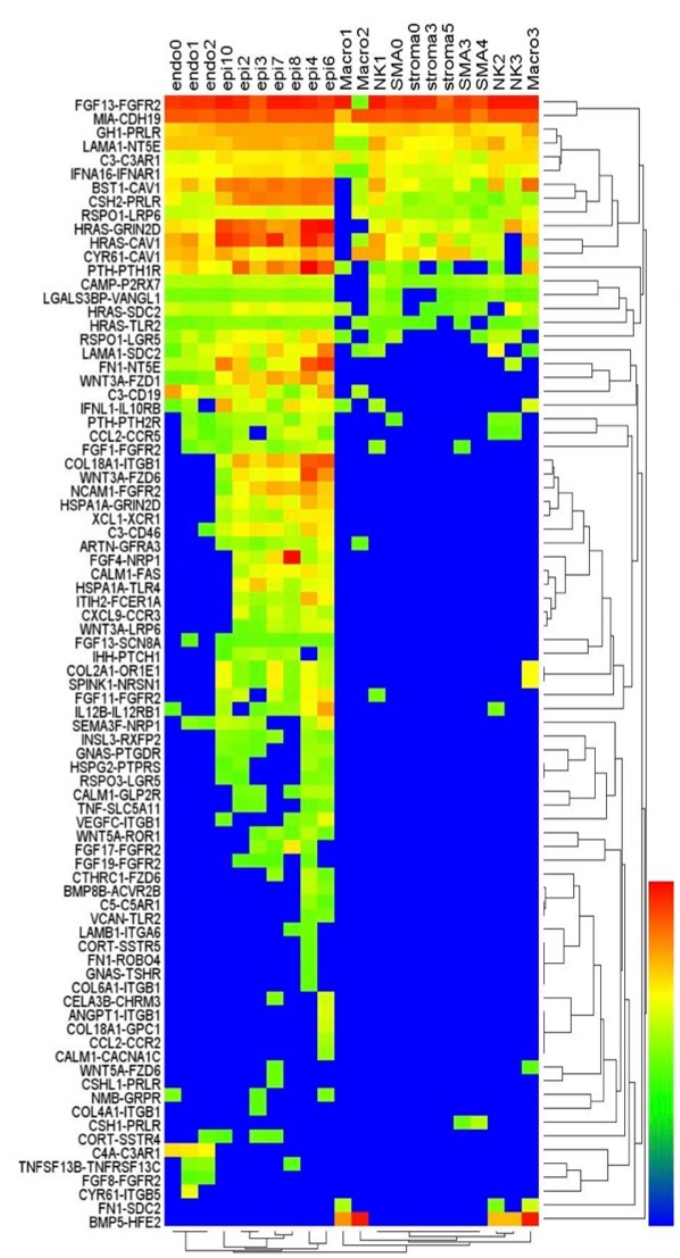

D

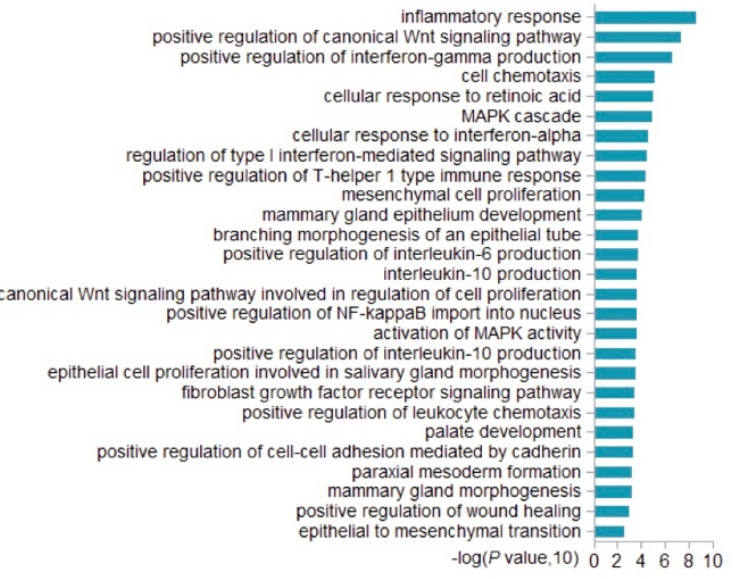




\section{Supplementary figures:}

Figure S1 Quality control of the single cell RNA-seq. (A) We used the Cell Ranger Pipeline (10x Genomics) to analyze the unique molecular tagged (UMI) of the raw sequencing data. (B) We collected 2735 cells with high UMI counts of the human uterus. (C) We obtained saturated sequencing with about 680k reads per cell. (D) The median gene number detected per cell was about 3,183.(E)\&(F) Unsupervised clustering based on principal components of the most variable expressed genes partitioned all the cells into 15 clusters, which was visualized with principal component analysis (PCA). Figure S1 was correlated with Figure 1.

Figure S2 Pseudospace ordering of the uterus epithelia subsets. (A) Pseudospace ordering of all the epithelial subclusters. (B) Order of each epithelial sub-cluster in the pseudospace (Epi-Epi6-Epi4-Epi5-Epi2-Epi3-Epi1-Epi0-Epi8-Epi7-Epi9). Figure S2 was correlated with Figure 2.

Figure S3 Gene ontology analysis of uterus epithelia subsets. (A) Gene ontology analysis of the highly expressed genes in the epithelial sub-cluster Epi2. (B) Gene ontology analysis of the highly expressed genes in the epithelial sub-cluster Epi3. (C) Gene ontology analysis of the highly expressed genes in the epithelial sub-cluster Epi4. (D) Gene ontology analysis of the highly expressed genes in the epithelial sub-cluster Epi5. (E) Gene ontology analysis of the highly expressed genes in the epithelial sub-cluster Epi6. (F) Gene ontology analysis of the highly expressed genes in the epithelial sub-cluster Epi7. (G) Gene ontology analysis of the highly expressed genes in the epithelial sub-cluster Epi8. (H) Gene ontology analysis of the highly expressed genes in the epithelial sub-cluster Epi9. Figure S3 was correlated with Figure 2.

Figure S4 Pseudospace ordering of the uterus stroma subsets. (A) Pseudospace ordering of all the stromal subclusters. (B) Order of each stromal sub-cluster in the pseudospace (stroma3-stroma2-stroma4-stroma0-stroma5-stroma1). Figure S4 was correlated with Figure 3.

Figure S5 Pseudospace ordering of the uterus endothelial subsets. (A) Pseudospace ordering of all the endothelial subclusters. (B) Order of each endothelial 
bioRxiv preprint doi: https://doi.org/10.1101/267849; this version posted February 19, 2018. The copyright holder for this preprint (which

was not certified by peer review) is the author/funder. All rights reserved. No reuse allowed without permission.

sub-cluster in the pseudospace (Endo4-Endo3-Endo1-Endo2-Endo0). Figure S5 was correlated with Figure 4. 


\section{Figure S1:}

A

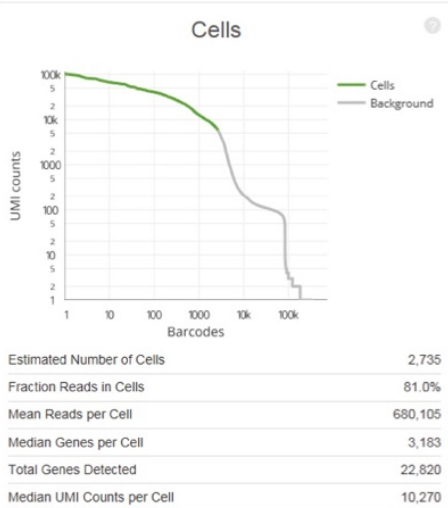

B

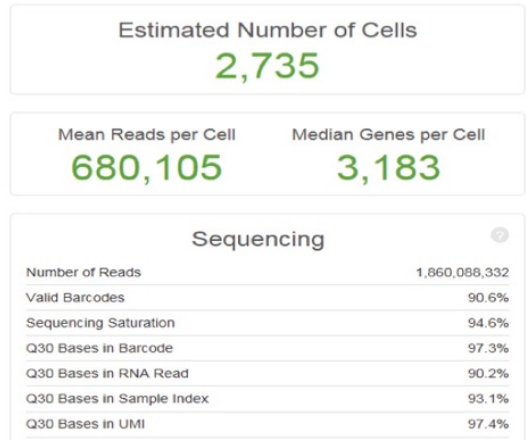

$\mathrm{E}$

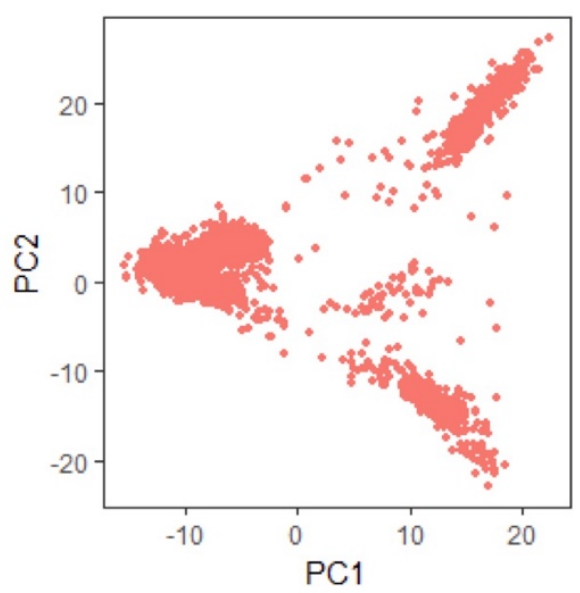

C

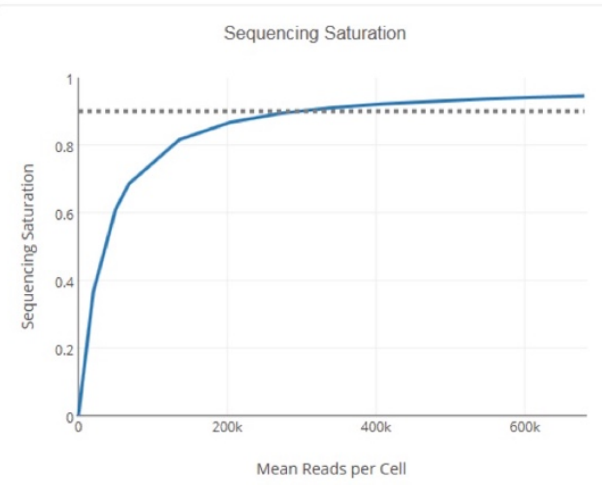

D

\section{nGene}

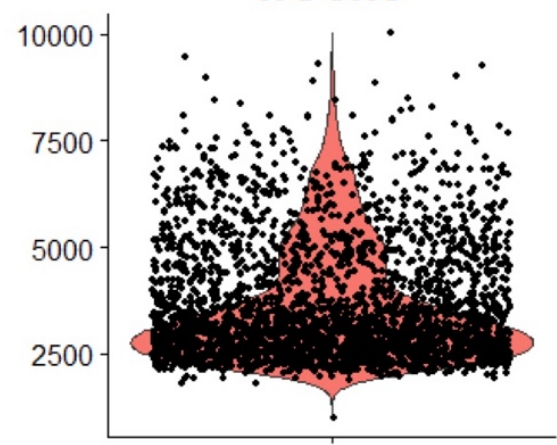

$\mathbf{F}$

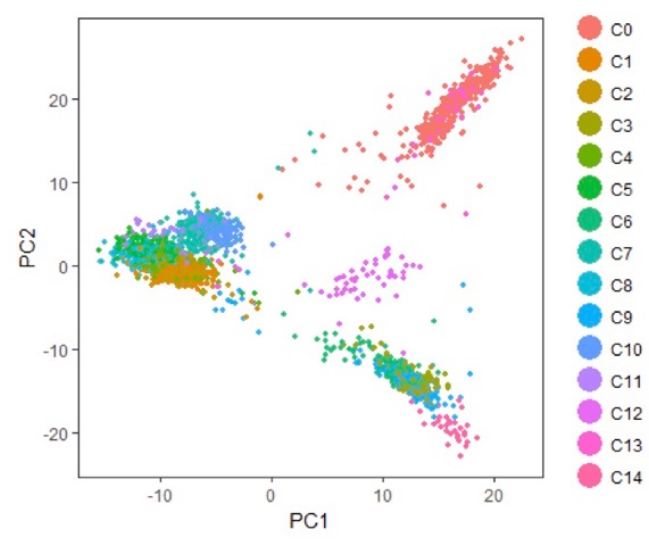




\section{Figure S2:}

A

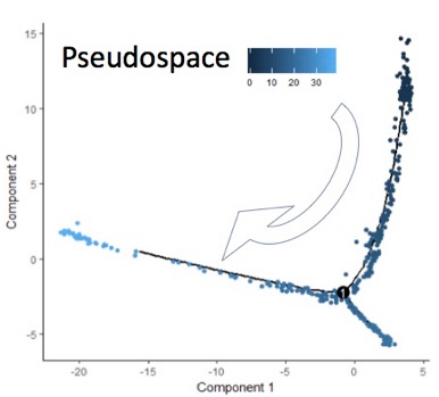

B

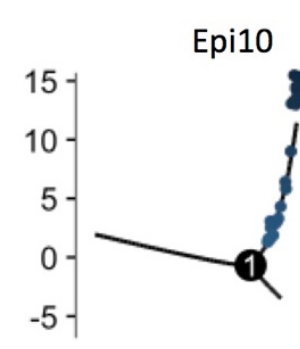

Epi6

Epi4

Epi5

Epi2

Epi3
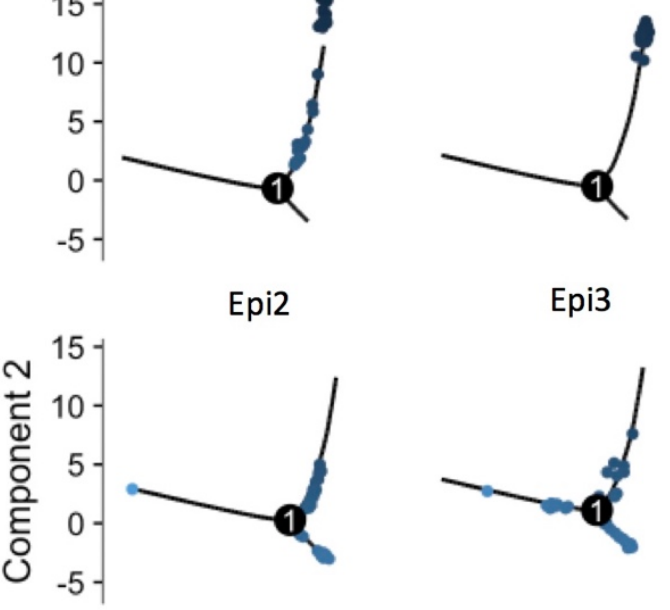

Epi8

$\begin{array}{r}15 \\ 10 \\ 5 \\ - \\ 0 \\ -5\end{array}-$

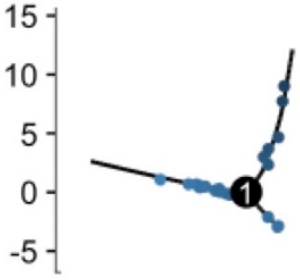

Epi7
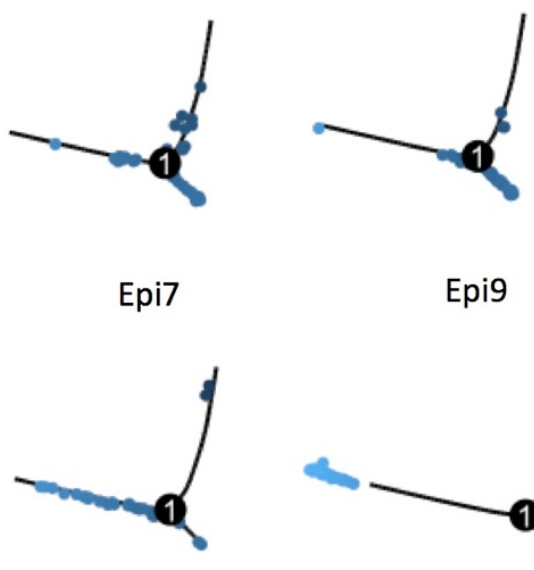

Epi9

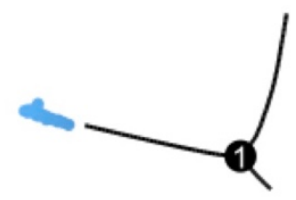

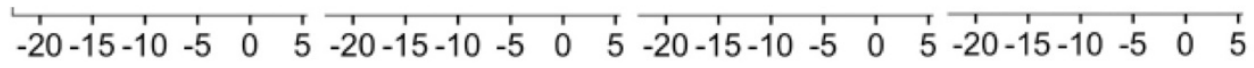

Component 1 
bioRxiv preprint doi: https://doi.org/10.1101/267849; this version posted February 19, 2018. The copyright holder for this preprint (which

was not certified by peer review) is the author/funder. All rights reserved. No reuse allowed without permission.

\section{Figure S3:}

A

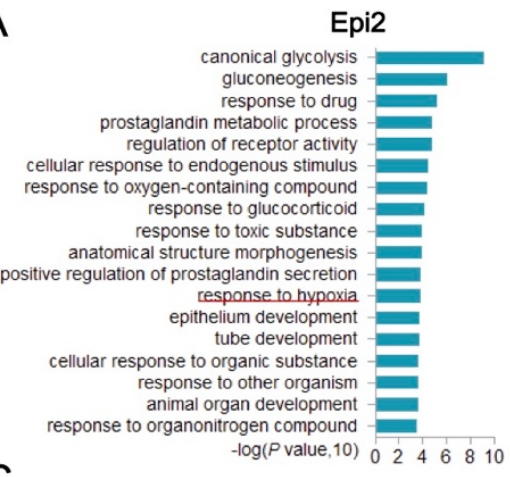

Epi2

canonical glycolysis gluconeogenesis rostaglandin metabolic process regulation of receptor activity cellular response to endogenous stimulus response to oxygen-containing compound
response to glucocorticoid response to toxic substance anatomical structure morphogenesis positive regulation of prostaglandin secretion response to hypoxia
epithelium development cellular response to organic substance response to other organism response to organonitrogen compound $\begin{array}{llllllll}-\log (P \text { value }, 10) & 0 & 2 & 4 & 6 & 8 & 10\end{array}$

C

regulation of transcription involved in G1/S transition of mitotic cell cycle DNA damage response detection of DNA damage DNA replication initiation nucleotide-excision repair DNA gap filling telomere maintenance via semi-conservative replication
CENP-A containing nucleosome assembly DNA replication-dependent nucleosome assembly sister chromatid cohesion error-free translesion synthesis DNA unwinding involved in DNA replication positive regulation of mitotic cell cycle phase transition transcription-coupled nucleotide-excision repair mismatch repair
positive regulation of chromosome segregation positive regulation of mitotic cross-link repair DNA ligation involved in DNA repair
Dution positive regulation of gene expression epigenetic double-strand break repair via homologous recombination DNA replication preinitiation complex assembly kinetochore organization

E $\begin{array}{lllll}-\log (P \text { value }, 10) & 0 & 5 & 10 & 15\end{array}$

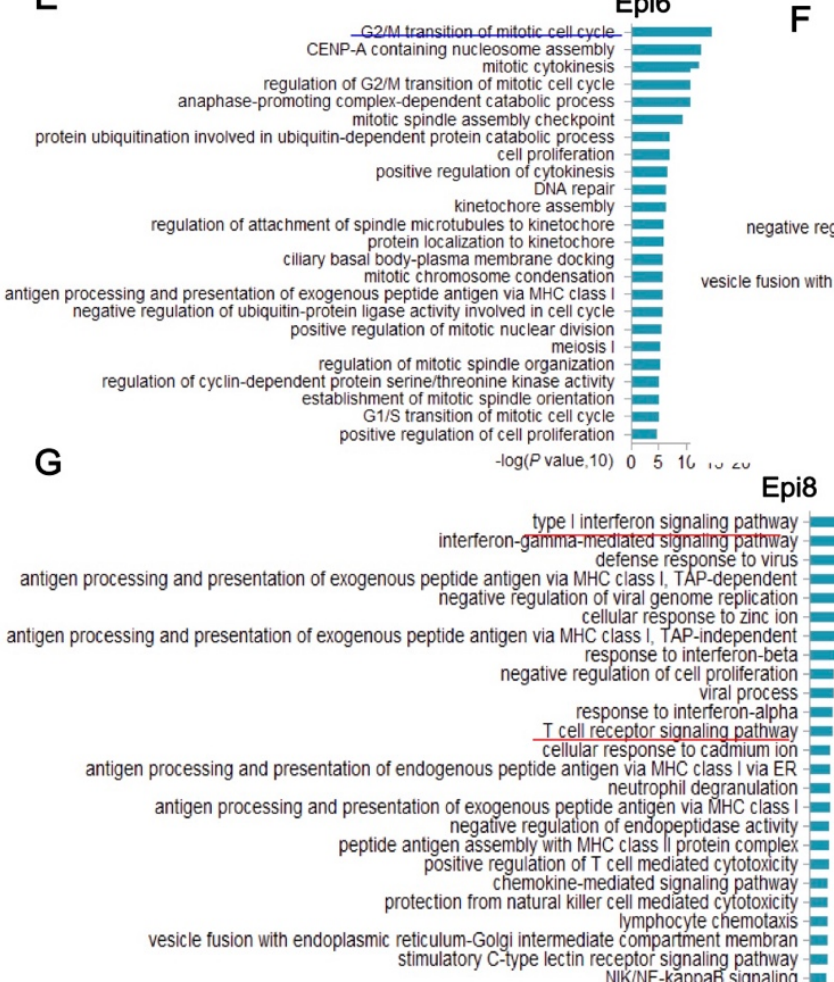
$-\log (P$ value 10$) \quad 0 \quad 10$
B

Epi3

positive regulation of cell proliferation
psitive regulation of transcription from RNA polymerase II promoter in response to stress

positive regulation of neutrophil chemotaxis -

prostaglandin biosynthetic process
regulation of receptor activity

response to glucocorticoid

cellular response to hypoxia

negative regulation orcell prollereration gonad development
gontic response angiogenesis
cell proliferation

mammary gland epithelial cell proliferation
cellular response to organic cyclic compound

response to vitamin
negative regulation of fat cell differentiation

negatve regula on or proen knase activity inflammatory response
endoplasmic reticulum unfolded protein response
response to activity

mitochondrial electron transport cytochrome $c$ to oxygen
regulation of inflammatory response

gative regulation of hormone secretion
positive regulation of cell proliferation

A polymerase II promoter in response to stress
negative regulation of immune system process
apoptotic process

$\begin{array}{lllllll}\text { apoptotic process } & & & & & & \\ -\log (P \text { value, } 10) & 0 & 2 & 4 & 6 & 8\end{array}$

Epi5

mRNA splicing via spliceosome

positive regulation of DNA biosynthetic process legative regulation of transcription DNA-templated mRNA transport

positive regulation of translational termination translational frameshifting viral process

chromatin organization DNA conformation change

nucleocytoplasmic transport

positive regulation of translational elongation

regulation of mRNA metabolic process $-\log (P$ value, 10$)$

Epi7

cellular response to zinc ion cellular response to cadmium ion protein transport -

peptide antigen assembly with MHC protein complex nucleoside phosphate catabolic process gative regulation of ER stress-induced intrinsic apoptotic signaling pathway negative regulation of growth mediate compartment membran regulation of biological quality ATF6-mediated unfolded protein response oxidation-reduction process cellular response to endogenous stimulus iron ion transport -

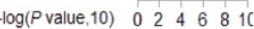

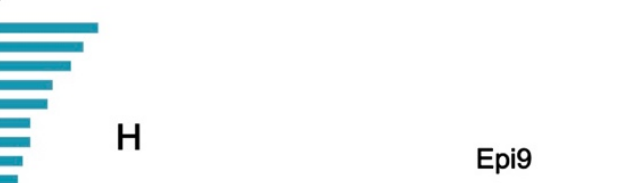

motile cilium assembly motile cilium assembly ciliary basal body-plasma membrane docking outer dynein arm assembly inner dynein arm assembly smoothened signaling pathway detection of chemical stimulus regulation of cilium assembly cilium movement involved in cell motility regulation of cilium movement $-\log (P$ value, 10$) 0 \quad 5 \quad 10 \quad 15 \%$ 


\section{Figure S4:}

A

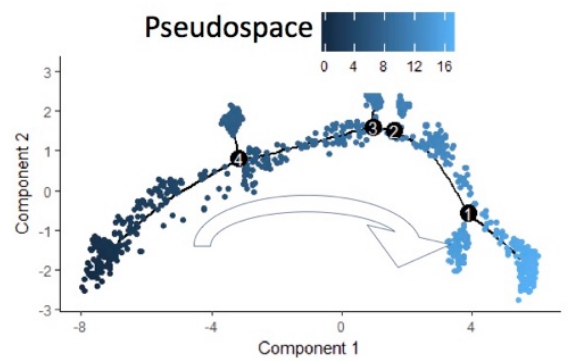

B

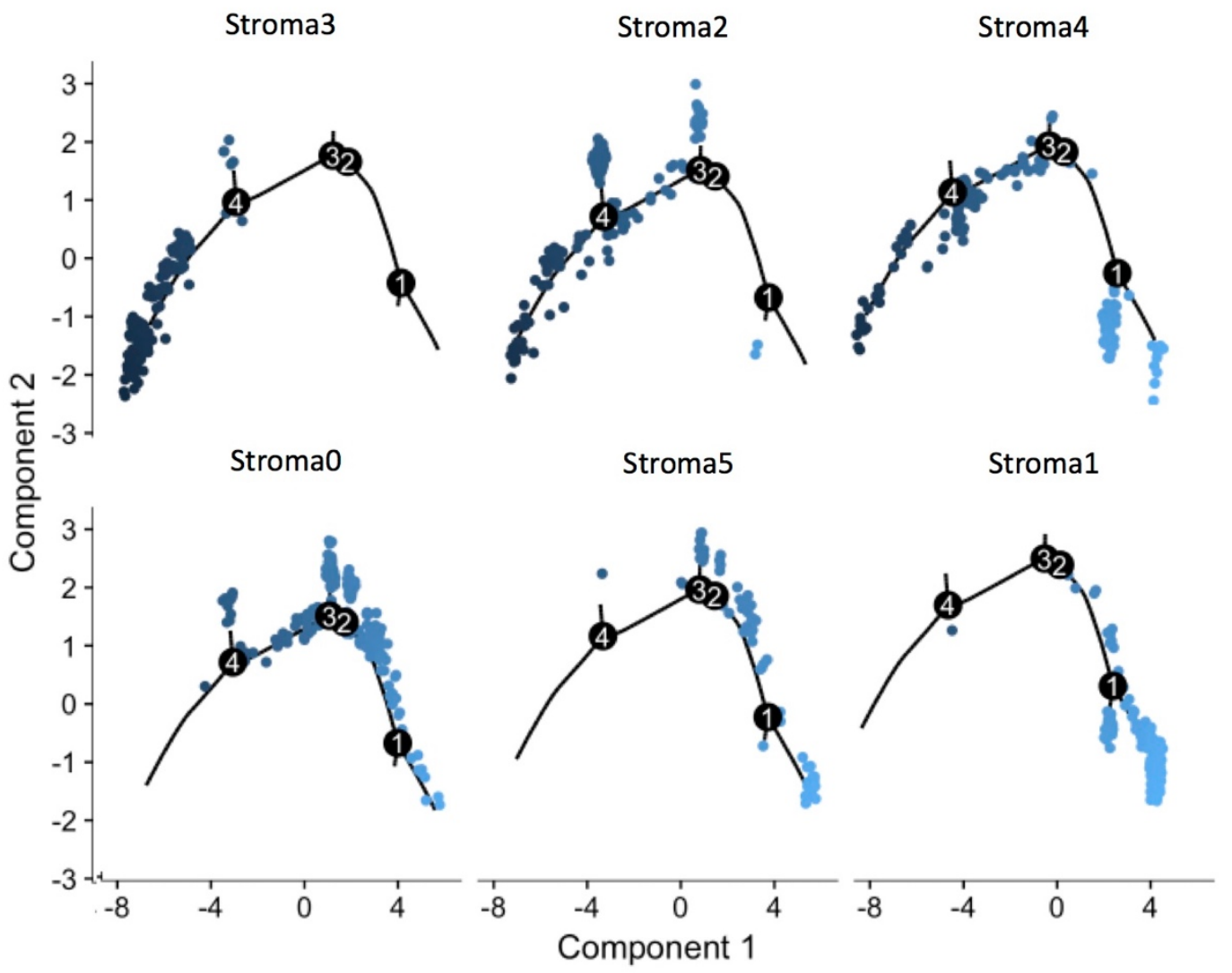




\section{Figure S5:}

A

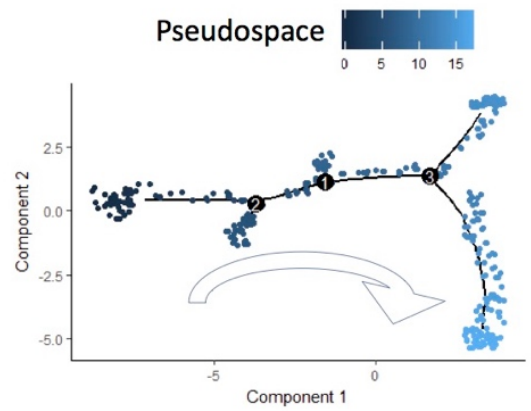

B

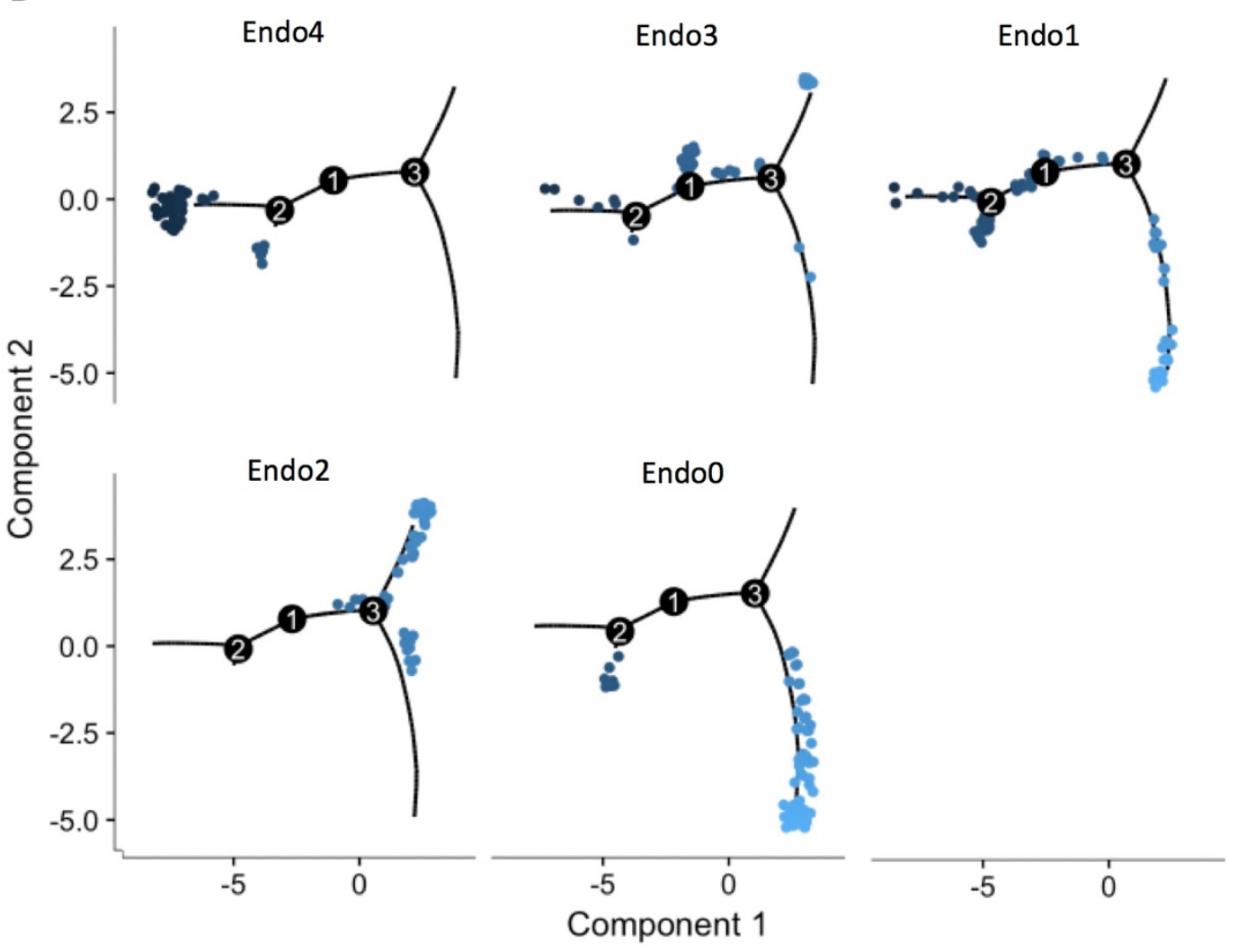

